\title{
Mimicking Hierarchical Complexity of the Osteochondral Interface Using Electrospun Silk-Bioactive Glass Composites
}

\author{
Joseph Christakiran M, ${ }^{\dagger}$ Philip J. T. Reardon, ${ }^{\dagger}$ Rocktotpal Konwarh, ${ }^{\dagger}$ Jonathan C. Knowles, ${ }^{*}$ \\ and Biman B. Mandal* ${ }^{\dagger} \dagger$ \\ ${ }^{\dagger}$ Biomaterial and Tissue Engineering Laboratory, Department of Biosciences and Bioengineering, Indian Institute of Technology \\ Guwahati, Guwahati 781039, Assam, India \\ ${ }^{\ddagger}$ Division of Biomaterials and Tissue Engineering, UCL Eastman Dental Institute, University College London, 256 Gray’s Inn Road, \\ London WC1X 8LD, U.K.
}

\section{Supporting Information}

ABSTRACT: The anatomical complexity and slow regeneration capacity of hyaline cartilage at the osteochondral interface pose a great challenge in the repair of osteochondral defects (OCD). In this study, we utilized the processing feasibility offered by the sol derived $70 \mathrm{~S}$ bioactive glass and silk fibroin (mulberry Bombyx mori and endemic Indian nonmulberry Antheraea assama), in fabricating a well-integrated, biomimetic scaffolding matrix with a coherent interface. Differences in surface properties such as wettability and amorphousness between the two silk groups resulted in profound variations in cell attachment and extracellular matrix

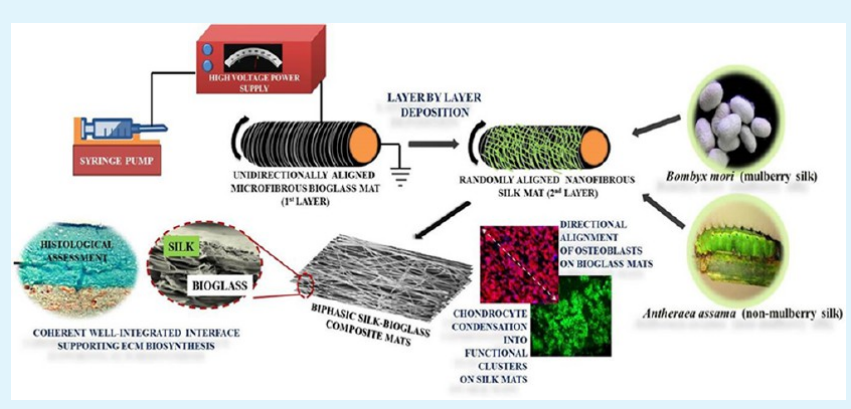
protein deposition. Mechanical assessment showed that the biphasic composites exhibited both an elastic region pertinent for cartilage tissue and a stiff compression resistant region simulating the bone phase. In vitro biological studies revealed that the biphasic mats presented spatial confinement for the growth and maturation of both osteoblasts and chondrocytes, marked by increased alkaline phosphatase (ALP) activity, osteopontin (OPN), sulfated glycosaminoglycan (sGAG) and collagen secretion in the cocultured mats. The non-mulberry silk based biphasic composite mats performed better than their mulberry counterpart, as evidenced by enhanced expression levels of key cartilage and bone specific marker genes. Therefore, the developed biphasic scaffold show great promise for improving the current clinical strategies for osteochondral tissue repair.

KEYWORDS: biomaterials, silk fibroin, nonmullberry silk, bioactive glass, osteochondral tissue engineering

\section{INTRODUCTION}

The number of orthopedic surgeries is on the rise and it is predicted to double globally by $2030 .^{1}$ In India alone, 70000 joint replacement surgeries were performed in $2011 .^{2}$ This scenario has necessitated innovative and affordable strategies in orthopedic care and management, particularly for defects at the osteochondral interface (OI). The OI is comprised of an anisotropic gradient of extracellular matrix and constituent cells, which includes a superficial hyaline cartilage layer, trailed by the middle transitional zone, followed by the deep zone which is in contact with the calcified subchondral bone. Osteochondral defects (OCD), a consequence of exposure of the subchondral bone, ${ }^{3}$ if left untreated can cause pain, swelling, and eventually limited range of motion and osteoarthritis. ${ }^{4}$ Generally, the surgical intervention for these defects utilizes reparative techniques such as autologous chondrocyte implantation (ACI), matrix assisted chondrocyte implantation (MACI) or mosaicplasty. ${ }^{5}$ However, these procedures often cause fibrocartilage to have poor resistance to shear and clinical durability and are restricted by the availability of donor tissue and donor site morbidity. Recently, tissue engineered constructs have continued to gain importance for treating bone and cartilage degeneration. $^{6-9}$ However, a gold standard material which structurally, mechanically and biologically fulfils the criteria for use in OCD repair is still required. An ideal OCD scaffold must possess a chondrogenic matrix that should be flexible, resilient and possess pores small enough to mimic the hyaline cartilaginous matrix; and an osteogenic matrix that should be mechanically competent and bioactive, possessing larger pores mimicking the microenvironment of the subchondral bone. ${ }^{10}$

Among the different strategies studied in recent years, biphasic structures developed from a natural polymer with suitable matrices to support both osteogenic and chondrogenic cells allowing a stable transition zone at the interface, have gained great interest. Silk fibroin (SF) based biomaterials have gained prominence in tissue engineering finding wide scale applications because of their superior cell supportive capability, excellent mechanical properties, tunable degradability and

Received: December 26, 2016

Accepted: February 9, 2017

Published: February 9, 2017 
versatile processability attributes. ${ }^{6,11}$ In recent years, silk based biomaterials for cartilage tissue engineering are gaining importance, as they present a conducive environment for maintaining the chondrogenic phenotype of seeded chondrocytes, while enabling enhanced extracellular matrix (ECM) secretion. $^{12,13}$ Moreover, nanofibrous electrospun silk matrices also exhibit high surface area appropriate for maximal cellmatrix interaction, in addition to conserving the elasticity of silk fibroin which is crucial for cartilage tissue engineering. ${ }^{14}$ The nanofibrous silk matrix thus provides the essential platform for cell condensation and cell-cell interaction that is required for chondrogenic phenotype maintenance. Furthermore, bioactive glasses continue to play a pivotal role in bone tissue engineering (BTE) due to their ability to stimulate more regeneration than any other ceramic applied for BTE applications. ${ }^{15}$ In particular, sol-gel derived glasses have received considerable research impetus because of their processing benefits over melt derived glasses, such as consistent purity, low temperature and reduced number of processing steps, while maintaining bioactivity. ${ }^{16}$ It has been demonstrated recently that electrospinning is an attractive method for the large scale production of consistent fibers that mimic the physico-chemical milieu of native ECM. The flexibility in processing afforded by sol-gel derived bioactive glasses and SF makes them ideal candidates for electrospinning. Thereby offering the exciting potential for producing replicable commercial scaffolds, this remains an elusive task for treatment in OCD repair.

Consequently, the purpose of the current study is to develop an electrospun composite scaffolds consisting of two separate phases, one capable of supporting the osteogenic precursor cells, and the other conducive to chondrogenic precursor cells, that are well integrated at the interface. To achieve this, we chose $70 \mathrm{~S}$ bioactive glass $\left(70 \mathrm{SiO}_{2} 25 \mathrm{CaO} .5 \mathrm{P}_{2} \mathrm{O}_{5}\right)$ as the osteogenic matrix, previously reported as an excellent candidate for BTE applications owing to its unique bioactivity, ${ }^{17}$ combined with silk fibroin from mulberry (Bombyx mori) and endemic northeast Indian non-mulberry (Antheraea assama) varieties to act as the chondrogenic matrix. These two silk varieties exhibit compositional diversity in the amino acids sequence. Recent reports on the non-mulberry silk have shown that the presence of RGD (arginine-glycine-aspartate) tripeptide and poly alanine repeats confer unique cell supportiveness and mechanical resilience to the SF matrices, respectively. ${ }^{18,19}$ In this context, mulberry and non-mulberry SF were investigated to scrutinize their effect as a suitable chondrogenic matrix. The biphasic constructs were fully characterized for their physico-chemical properties such as structural conformation, wettability, degradation and swelling behavior. Furthermore, the ability of the biphasic constructs to synergistically support the growth of chondrocytes and osteoblasts was evaluated by coculturing porcine auricular chondrocytes and a human osteoblast cell line (MG63) as a model system; and the cellular, biochemical and gene expression profile were studied to assess the suitability of the constructs as potential matrices for OCD repair.

\section{EXPERIMENTAL SECTION}

2.1. Materials. Calcium nitrate tetrahydrate (Sigma-Aldrich), Butvar-B98 (polyvinyl-butyrate) - PVB (Sigma-Aldrich), poly(vinyl alcohol) - PVA (Himedia, India), tetraethyl-orthosilicate (TEOS) (Sigma-Aldrich), triethyl phosphate (TEP) (Sigma-Aldrich), hydrochloric acid (Merck, India), ethanol (Jiangsu Huaxi Int. Ltd., China), alamar blue (Invitrogen), alkaline phosphatase assay kit (Abcam, U.K.), calcein-AM and ethidium homodimer (Sigma-Aldrich).

2.2. Methods. 2.2.1. Fabrication of the Bilayered Composites. Synthesis of 705 Bioactive Glass and Electrospinning of 705 Bioactive Glass. The $70 \mathrm{~S}$ bioactive glass was synthesized following a previously published protocol. ${ }^{17}$ Briefly, TEOS, calcium nitrate tetrahydrate and TEP were added in the molar ratio of 70:25:5 in ethanol/water solvent system with $2 \%(\mathrm{v} / \mathrm{v}) \mathrm{HCl}$ in the molar ratio of 1:2:2 TEOS/ethanol/water. The sol obtained was aged for $48 \mathrm{~h}$ at 40 ${ }^{\circ} \mathrm{C}$. The aged sol was mixed with $10 \%(\mathrm{w} / \mathrm{v})$ PVB prepared in absolute ethanol (to enhance the rheological properties of the sol) in the ratio of $1: 4(\mathrm{v} / \mathrm{v})$. The solution was electrospun using a blunt $21 \mathrm{G}$ needle in an electrospinning setup (E-spin nanotech, India) at a voltage of 16 $\mathrm{kV}$, a working distance of $10 \mathrm{~cm}$ and a flow rate of $1 \mathrm{~mL} / \mathrm{h}$ and the fibers were collected over a rotating mandrel (of diameter $40 \mathrm{~mm}$ and length $165 \mathrm{~mm}$, rotational speed of $1550 \mathrm{rpm}$ ) at ambient conditions. The obtained bioactive glass (BG) mats were dried overnight at room temperature to remove residual solvent.

Isolation of Silk Protein and Electrospinning of Silk. For isolation of mulberry silk, B. mori cocoons were obtained from local silk farms and the cocoons were processed based on a previously published method. ${ }^{20}$ Briefly, the cocoons were cut into small pieces and degummed in boiling $0.02 \mathrm{M} \mathrm{Na}_{2} \mathrm{CO}_{3}$ and fibers obtained were dried at room temperature. The dried, degummed silk fibers were dissolved in $9.3 \mathrm{M} \mathrm{LiBr}$ (Sigma-Aldrich), followed by subsequent dialysis done extensively using a $12 \mathrm{kDa}$ cutoff dialysis membrane (Sigma-Aldrich) against distilled water for $48 \mathrm{~h}$. The regenerated aqueous silk fibroin solution was further used for electrospinning. For isolation of nonmulberry silk, mature fifth instar A. assama silkworms were obtained from local silk farms. The glandular protein was isolated using a previously published protocol. ${ }^{21}$ Briefly, the non-mulberry silk was squeezed out from silk glands of fifth instar A. assama silkworms and the silk protein was dissolved using $1 \%(\mathrm{w} / \mathrm{v})$ sodium dodecyl sulfate (SDS) (Himedia, India) followed by its extensive dialysis at $4{ }^{\circ} \mathrm{C}$. The regenerated aqueous silk solution was further used for electrospinning.

The B. mori silk (3\% w/v) was blended with PVA (to improve the rheological property of the silk solution) $(13 \% \mathrm{w} / \mathrm{v})$ in the ratio $1: 1$ $(\mathrm{v} / \mathrm{v})$ and $10 \mathrm{~mL}$ of the blended solution was electrospun using a blunt $21 \mathrm{G}$ needle, at a voltage of $21 \mathrm{kV}$, at a working distance of $13 \mathrm{~cm}$ and a flow rate of $1 \mathrm{~mL} / \mathrm{h}$ to obtain the B. mori silk mats (BM). Similarly $A$. assama silk $(3 \% \mathrm{w} / \mathrm{v})$ was blended with PVA $(13 \% \mathrm{w} / \mathrm{v})$ in the ratio $1: 1(\mathrm{v} / \mathrm{v})$ and $10 \mathrm{~mL}$ of the blended solution was electrospun using a blunt $21 \mathrm{G}$ needle, at a voltage of $21 \mathrm{kV}$, at a working distance of 13 $\mathrm{cm}$, mandrel rotational speed of $1550 \mathrm{rpm}$, and a flow rate of $1 \mathrm{~mL} / \mathrm{h}$ at ambient conditions to obtain the A. assama silk mats (AA).

Electrospinning of Bilayered Composite Mats. For obtaining the composite bilayered mats, a layer by layer approach was followed, wherein the bioactive glass (volume $=5 \mathrm{~mL}$ ) was spun over the mandrel, the silk (volume $=5 \mathrm{~mL}$ ) was spun over the top of the spun bioactive glass mats (with parameters maintained as mentioned previously). Thus, two mats were obtained namely PVB-bioactive glass/PVA - B. mori silk (BI) and PVB-bioactive glass/PVA - A. assama (AI) silk which were further evaluated for their cytocompatibility. All the mats were then treated with absolute ethanol for $10 \mathrm{~min}$, followed by $70 \%(\mathrm{v} / \mathrm{v})$ ethanol for $10 \mathrm{~min}$ and vacuum-dried to induce crosslinking and also to confer insolubility within the silk matrices.

2.2.2. Physico-Chemical Characterizations. Scanning Electron Microscopy (SEM). Analysis of fiber diameter and morphology was carried out using scanning electron microscopy (XL30 FEG, Philips, Netherlands). SEM micrographs were analyzed using Image-J (Wayne Rasband, National Institute of Health) to determine the average diameter and standard deviation of the population of fibers ( 50 fibers were measured from each sample).

Fourier Transform Infrared (FTIR) Spectroscopy. The infrared spectra of all electrospun samples were recorded using an FTIR-ATR (PerkinElmer 2000 spectrophotometer). Sliced samples of the mats were placed on the ATR crystal, and then compressed using an axial screw. Spectra of all samples were recorded using a frequency range between 400 and $4000 \mathrm{~cm}^{-1}$, and averaged over 4 runs. 
$X$-ray Diffraction. The X-ray diffraction (XRD) patterns of samples were obtained from a Bruker D8 Advance X-ray diffractometer with a $\mathrm{CuK}_{\alpha}(\lambda=0.1541784 \mathrm{~nm})$ radiation source. Diffraction patterns were collected from $10^{\circ}$ to $60^{\circ}$ with a step size of $0.02^{\circ}$ and $1 \mathrm{~s}$ per step was used.

Contact Angle Measurements. Static contact angle measurements were performed on dry films $(n=3)$ using a goniometer (CAM200, $\mathrm{KSV}$, Sweden). Ultrapure distilled water droplets were used for measurements. Contact angle measurements were taken for monophasic electrospun mats (BG, BM, and AA) and for biphasic composite mats (BI and AI) on bioactive glass side and silk fibroin side. The measurements are represented as mean \pm standard deviation.

Mechanical Testing. The tensile mechanical properties of the electrospun fiber mats were recorded using Universal Testing Machine (Instron, model: 5944) equipped with a $100 \mathrm{~N}$ load cell at a crosshead rate of $1 \mathrm{~mm} / \mathrm{min}$. Electrospun mats were cut into rectangular strips of $1 \mathrm{~cm} \times 3 \mathrm{~cm}$, as defined in ASTMD882-02. The samples were mounted in silicon carbide paper grips prior to placement in pneumatic grips. Measurements were carried out in triplicate under ambient conditions. The Young's Modulus was calculated using an offset-yield approach. ${ }^{22}$ A line was drawn parallel to the linear regression elastic region at an offset of $0.5 \%$ to the initial sample gauge length; the intersection point wherein the line met the stress-strain curve was defined as the Young's modulus for the sampling.

Swelling Properties. Swelling percentage was evaluated using previously published protocols. ${ }^{23}$ Briefly, electrospun mats $(n=4)$ of predetermined weight $\left(W_{\mathrm{D}}\right)$ were immersed in phosphate buffered saline (PBS; $\mathrm{pH} 7.4)$ and at regular intervals swollen mats were weighed $\left(W_{\mathrm{S}}\right)$ after wicking off excess PBS using filter paper. The swelling percentage was calculated by applying the following equation:

$$
\text { swelling percentage } \left.(\%)=\left(\left(W_{\mathrm{S}}-W_{\mathrm{D}}\right) / W_{\mathrm{D}}\right)\right) \times 100
$$

In Vitro Enzymatic Degradation. The in vitro enzymatic degradation was carried out in the presence of protease XIV (SigmaAldrich, $\geq 3.5 \mathrm{U} / \mathrm{mg}$; isolated form Streptomyces griseus) according to earlier reports. ${ }^{12}$ Briefly, the electrospun mats $(n=4)$ of predetermined weight were immersed in PBS ( $\mathrm{pH} 7.4$ ) with $2 \mathrm{U} /$ $\mathrm{mL}$ protease $\mathrm{XIV}$ at $37^{\circ} \mathrm{C}$. At regular intervals the mats were retrieved, washed with PBS ( $\mathrm{pH}$ 7.4) and dried. The mass remaining was recorded and the following equation was implied to calculate the mass remaining:

$$
\% \text { mass remaining }=\left(\text { mass at time }{ }^{\prime} t^{\prime} / \text { initial mass }\right) \times 100
$$

Protein Adsorption Studies. Protein adsorption studies were carried out using bovine serum albumin (BSA) (Sigma-Aldrich) following previously reported protocols. ${ }^{24}$ Briefly, electrospun mats (10 mm diameter) were immersed in PBS ( $\mathrm{pH} 7.4$ ) with $20 \mathrm{mg} / \mathrm{mL}$ BSA for $24 \mathrm{~h}$. The protein concentration in solution after incubation was estimated using Bradford's reagent (Sigma-Aldrich) and the amount of protein adsorbed was estimated by subtracting from the initial protein solution. A calibration curve was plotted taking BSA as standard for protein concentration estimation.

2.2.3. In Vitro Biological Studies. For cell culture, the mats $(10 \mathrm{~mm}$ diameter) were sterilized using $70 \%$ ethanol followed by UV irradiation for $20 \mathrm{~min}$. The sterile mats were placed in 24 well plate $(n=4)$ while cells grown in tissue culture plate (TCP) served as control. MG63 (osteosarcoma cell line, NCCS Pune) were maintained in high-glucose DMEM (Gibco) supplemented with 10\% FBS (Gibco). The porcine ear chondrocytes were isolated from the porcine pinna of pigs obtained from local slaughter house. The chondrocytes were isolated based on a previously published protocol. ${ }^{12}$ Briefly, bilateral ear cartilage was harvested post stripping the perichondrium, under sterile conditions. The obtained cartilage was diced and further subjected to enzyme digestion $(0.2 \%(\mathrm{w} / \mathrm{v})$ type XIV protease (Sigma-Aldrich, $\geq 3.5 \mathrm{U} / \mathrm{mg}$ ) for $1 \mathrm{~h}, 0.05 \%$ type I-A collagenase (Sigma-Aldrich, $\geq 125 \mathrm{CDU} / \mathrm{mg}$ ) overnight at $37^{\circ} \mathrm{C}, 95 \%$ relative humidity and $\left.5 \% \quad \mathrm{CO}_{2}\right)$. The resulting digestate was centrifuged at $2000 \mathrm{rpm}$ for $5 \mathrm{~min}$ and cell pellet was washed thrice with high glucose Dulbecco's modified Eagle's medium (DMEM, Gibco). The chondrocytes obtained were maintained in high glucose DMEM supplemented with $10 \%$ fetal bovine serum (FBS, Gibco).

In Vitro Cell Proliferation Assay. For the evaluation of cell proliferation the alamar blue (Invitrogen) assay was performed based on the manufacturer's protocol at $1,3,7,11$, and 14 days. Briefly the BG, BM, and AA mats were seeded with MG63-osteoblast at a density of $10^{5}$ cells per $10 \mathrm{~mm}$ membrane used $(n=4)$; similarly the $\mathrm{BG}, \mathrm{BM}$, and $\mathrm{AA}$ mats were seeded with primary chondrocytes at a seeding density of $10^{5}$ cells per $10 \mathrm{~mm}$ membrane used $(n=4)$. For the composite bilayered mats, the cells were cocultured. Initially the MG63 osteoblasts were seeded and on the bioactive glass side at a seeding density of $5 \times 10^{4}$ cells per $10 \mathrm{~mm}$ membrane, after $2 \mathrm{~h}$ the mats were flipped and primary porcine chondrocytes were seeded at a seeding density of $5 \times 10^{4}$ cells per $10 \mathrm{~mm}$ membrane $(n=4)$. The cell seeded membranes were incubated with $10 \%(\mathrm{v} / \mathrm{v})$ alamar blue dye in culture media for $3 \mathrm{~h}$. Post incubation, $100 \mu \mathrm{L}$ of the culture media was read at 570/600 nm using microplate reader (Tecan Infinite Pro, Switzerland). The results are represented as normalized alamar units at different time intervals. Subsequently, the cell seeded membranes were maintained for 14 days in culture in high glucose DMEM supplemented with $10 \% \mathrm{FBS}$ at $37{ }^{\circ} \mathrm{C}$ and $5 \% \mathrm{CO}_{2}$ with subsequent media change every $48 \mathrm{~h}$.

Live/Dead Imaging. The cell seeded mats $(n=4)$ after day 14 were visualized for the distribution of live and dead cells using calcein-AM and ethidium homodimer (Sigma-Aldrich). Briefly, the mats were washed with phosphate buffered saline (PBS, $\mathrm{pH}$ 7.4) and the mats were incubated at $37{ }^{\circ} \mathrm{C}$ and $5 \% \mathrm{CO}_{2}$ with $40 \mathrm{nM}$ calcein-AM and 20 $\mathrm{nM}$ ethidium homodimer for $20 \mathrm{~min}$. The dye mix was removed and washed twice with PBS and the mats were visualized under fluorescence microscope (EVOS XL Digital microscope) and representative images are presented.

Cytoskeletal Architecture Assessment. In order to visualize the cytoskeletal architecture of the cells seeded on the electrospun mats, the cell seeded mats were fixed with neutral buffered formalin (NBF) (Sigma-Aldrich). The fixed constructs were treated with $0.165 \mu \mathrm{M}$ phalloidin conjugated to rhodamine (Life technologies, U.S.A.) to stain the F-actin and counter stained with Hoechst-33342 (SigmaAldrich). The mats were then visualized under fluorescence microscope (EVOS XL Digital microscope) and representative images are presented.

Biochemical Analysis. Alkaline Phosphatase Assay. For determining the membrane bound alkaline phosphatase (ALP), MG63 cells were seeded on BG, BM and AA electrospun mats at a seeding density of $10^{5}$ cells per $10 \mathrm{~mm}$ membrane $(n=4)$ as control. Whereas in the bilayered composite membranes, chondrocytes and MG63 were cocultured $\left(5 \times 10^{4}\right.$ chondrocytes and $5 \times 10^{4}$ MG63 per $10 \mathrm{~mm}$ membrane $(n=4))$. At different time points the cells laden membranes were lysed using cell lysis buffer $(20 \mathrm{mM}$ Tris- $\mathrm{HCl}$ (Merck, India) (pH 7.5), $150 \mathrm{mM} \mathrm{NaCl}$ (Himedia, India), $5 \mathrm{mM}$ $\mathrm{MgCl}_{2}$ (Himedia, India), and 0.5\% Triton-X100 (Sigma-Aldrich). The ALP activity was determined following the manufacturer's protocol (Abcam, alkaline phosphatase assay kit (Abcam, U.K.). The ALP activity expressed as $\mathrm{U} / \mathrm{ml}$ was normalized with the total DNA content for both the membrane bound and soluble ALP and represented as U/ $\mu$ DNA. $^{25}$

Total Collagen Estimation. To determine the amount of collagen secreted by cells in response to the mats, BG, BM, and AA mats were seeded with MG63-osteoblast at a density of $10^{5}$ cells per $10 \mathrm{~mm}$ membrane used $(n=4)$; similarly the BG, BM, and AA mats were seeded with primary chondrocytes at a seeding density of $10^{5}$ cells per $10 \mathrm{~mm}$ membrane used $(n=4)$. For the composite bilayered mats, the cells were cocultured. MG63 Osteoblasts were seeded on the bioactive glass side at a seeding density of $5 \times 10^{4}$ cells per $10 \mathrm{~mm}$ membrane and primary porcine chondrocytes were seeded at a seeding density of $5 \times 10^{4}$ cells per $10 \mathrm{~mm}$ membrane $(n=4)$. A previously published protocol utilizing sirius red dye based colorimetric assay ${ }^{26}$ with rat tail collagen (Sigma-Aldrich) $(0-250 \mu \mathrm{g} / \mathrm{mL})$ as standard, was followed. The cell laden mats $(n=4)$ were digested with pepsin (Sigma-Aldrich, $1 \mathrm{mg} / \mathrm{mL} \mathrm{pH} \mathrm{3.0).} \mathrm{An} \mathrm{aliquot} \mathrm{of} 100 \mu \mathrm{L}$ from the digested sample was 
Table 1. Primer Sequences of Different Genes Used for Gene Expression Studies

\begin{tabular}{|c|c|c|}
\hline gene & sequence & accession number \\
\hline Human GAPDH & $\begin{array}{l}\text { F 5'-GACCTGACCTGCCGTCTA-3' } \\
\text { R 5'-GTTGCTGTAGCCAAATTCGTT-3' }\end{array}$ & NM_001289746.1 \\
\hline Human BSP & $\begin{array}{l}\text { F 5'AACCTACAACCCCACCACAA-3' } \\
\text { R 5'-GTTCCCCGTTCTCACTTTCA-3' }\end{array}$ & NM_004967.3 \\
\hline Human runx2 & $\begin{array}{l}\text { F 5'-GATGGGACTGTGGTTACTGTCA-3' } \\
\text { R 5'-CTCAGATCGTTGAACCTTGC-3' }\end{array}$ & NM_001278478.1 \\
\hline Porcine GAPDH & $\begin{array}{l}\text { F 5'-TCGGAGTGAACGGATTTGG-3' } \\
\text { R 5'-CCAGAGTTAAAAGCAGCCCT-3' }\end{array}$ & NM_001206359.1 \\
\hline Porcine aggrecan & $\begin{array}{l}\text { F 5'-CCCAACCAGCCTGACAACTT-3' } \\
\text { R 5'-CСТTCTCGTGCCAGATCATCA-3' }\end{array}$ & NM_001164652.1 \\
\hline Porcine sox-9 & $\begin{array}{l}\text { F 5'-TTCCGCGACGTGGACAT-3' } \\
\text { R 5'-GGCGGCAGGTACTGGTCAAACTC-3' }\end{array}$ & NM_213843.1 \\
\hline
\end{tabular}

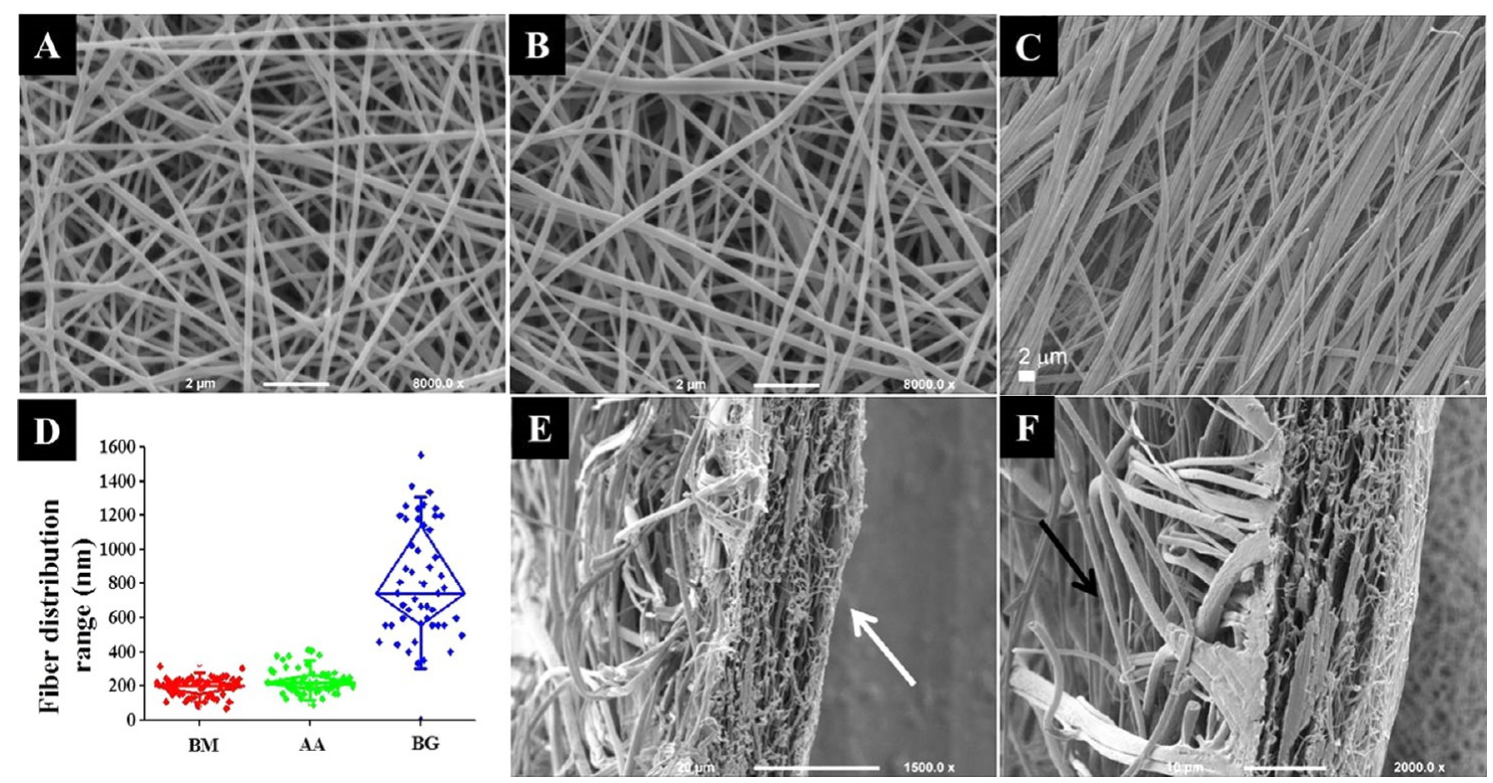

Figure 1. Scanning electron micrographs of (A) BM, (B) AA, and (C) BG mats; (D) fiber distribution analysis showing nanofibrous nature of SF mats and microfibrous nature of BG mats; SEM micrograph of a cross-section of biphasic mats formed by (E) electrospininning BG, followed by (F) silk layer, exhibiting coherent well integrated interface (white arrow indicating nanoporous SF layer and black arrow indicating microporous BG layer).

allowed to dry at $37^{\circ} \mathrm{C}$ in 96 well-plate overnight. The dried sample was treated with sirius red dye solution $(1 \mathrm{mg} / \mathrm{mL})$ saturated with picric acid for $1 \mathrm{~h}$, the samples were washed with $0.01 \mathrm{~N} \mathrm{HCl}$ thrice. Finally the samples were dissolved in $0.1 \mathrm{~N} \mathrm{NaOH}$ (Merck, India) and the absorbance were recorded at $550 \mathrm{~nm}$ and the collagen content was determined with reference to rat tail collagen as standard.

Sulfated Glycosaminoglycan Content. To determine the amount of collagen secreted by cells in response to the mats, BG, BM, and AA mats were seeded with primary porcine chondrocytes at a density of $10^{5}$ cells per $10 \mathrm{~mm}$ membrane used $(n=4)$. For the composite bilayered mats, the cells were cocultured. MG63 Osteoblasts were seeded on the bioactive glass side at a seeding density of $5 \times 10^{4}$ cells per $10 \mathrm{~mm}$ membrane and primary porcine chondrocytes were seeded at a seeding density of $5 \times 10^{4}$ cells per $10 \mathrm{~mm}$ membrane $(n=4)$. A previously published protocol was followed for sGAG estimation using 1,9-dimethylmethylene blue (DMMB) assay. ${ }^{27}$ Briefly the cell seeded membranes were digested using papain digestion solution $(125 \mu \mathrm{g} / \mathrm{mL}$ papain (Sigma-Aldrich), $5 \mathrm{mM}$ L-cysteine (Sigma-Aldrich), $100 \mathrm{mM}$ $\mathrm{Na}_{2} \mathrm{HPO}_{4}$ (Merck, India), $5 \mathrm{mM}$ Ethylenediaminetetraacetic acid (EDTA, Sigma-Aldrich)) at $60{ }^{\circ} \mathrm{C}$ for $16 \mathrm{~h}$. The amount of sulfated glycosaminoglycan was determined using DMMB (Sigma-Aldrich), with reference to chondroitin sulfate from bovine trachea (SigmaAldrich) as standard, by measuring the absorbance at $525 \mathrm{~nm}$ using Tecan infinite-pro microplate reader.
Gene Expression Studies. For analyzing the osteogenic and chondrogenic potential of cells cultured on the different mats, the relative gene expression was assessed after day 1 , day 7 , and day 14 for osteogenic genes namely bone sialoprotein (BSP), runt-related transcription factor 2 (runx2) and for chondrogenic genes namely aggrecan and sox-9. RNA was isolated by lysing the cells using TRIzol reagent (Sigma-Aldrich). The lysate was centrifuged at $13000 \mathrm{rpm}$ (10 min, $4{ }^{\circ} \mathrm{C}$ ) and the supernatant was transferred to fresh tubes. After incubation with chloroform for $15 \mathrm{~min}$, the mixture was centrifuged at $13000 \mathrm{rpm}\left(15 \mathrm{~min}, 4^{\circ} \mathrm{C}\right)$ and upper aqueous layer was transferred to new tubes. RNA obtained was further eluted, purified using ethanol and finally resuspended in RNase free water (Sigma-Aldrich). RNA was reverse transcribed using high capacity reverse transcription kit (Applied Biosystems, Invitrogen) in a thermal cycler machine (TaKaRa, Japan). Expression level of genes was quantified using Power SYBR Green PCR master mix (Applied Biosystems, Life technologies) in a real-time PCR machine (Applied Biosystems 7500) with the sequences shown in Table 1.

In Vitro Immune Response Assessment. In order to assess the immune response elicited by the mats $(n=4)$, murine macrophage cells (RAW 264.7, obtained from NCCS, Pune) were utilized. The TNF- $\alpha$ secreted by macrophages was quantified using an ELISA kit (Invitrogen) based on the manufacturer's protocol. Briefly, $10^{5}$ cells/ $\mathrm{cm}^{2}$ were seeded on 24 well plates, and after $24 \mathrm{~h}$ mats of diameter 10 
$\mathrm{mm}$ were placed on the seeded wells and the spent media supernatant after 12 and $24 \mathrm{~h}$ were collected and assayed for the TNF- $\alpha$ production. $500 \mathrm{ng}$ Lipopolysaccharides (LPS) from Escherichia coli (Sigma-Aldrich) served as the positive control, while tissue culture plate (TCP), wells without any samples served as negative control.

Histological Assessment and Immunostaining. The cell seeded membranes were fixed with NBF. The membranes were subjected to ethanol-xylene dehydration procedure and embedded in paraffin and sectioned using manual rotary microtome (Leica biosystems.) to obtain $10 \mu \mathrm{m}$ thick slices. The slices were further stained with hematoxylin and eosin to observe cell-scaffold interaction and the distribution of cells on and within the membrane. The sections were stained with $2 \%$ alizarin red (Sigma-Aldrich) to assess the extent of the calcium deposition and 1\% alcian blue (Sigma-Aldrich) to determine the extent of sulfated glycosaminoglycan deposition. For immunostaining, cell seeded electrospun mats were fixed with NBF overnight. The fixed constructs were permeabilized with $0.1 \%$ Triton X-100 (SigmaAldrich) in PBS for $15 \mathrm{~min}$, followed by blocking with $1 \%$ BSA (Sigma-Aldrich) in PBS. The mats were incubated with corresponding primary antibody, rabbit polyclonal against collagen-II (Abcam, U.K., 1:200 dilution) for chondrocytes and rabbit polyclonal against osteopontin (OPN) (Abcam, U.K., 1:1000 dilution) for osteoblasts, overnight at $4{ }^{\circ} \mathrm{C}$. The mats were then incubated with FITC conjugated secondary antibody antirabbit developed in goat (Abcam, U.K., 1:2000) for $1 \mathrm{~h}$ at room temperature. The mats were counterstained with $0.165 \mu \mathrm{M}$ phalloidin conjugated to rhodamine (Life technologies) to stain the F-actin and with Hoechst-33342 (Sigma-Aldrich) to stain the nucleus. At each step the mats were washed with $0.1 \%$ Tween-20 (Sigma-Aldrich) in PBS. The stained sections or mats were visualized using inverted fluorescence EVOS XL Digital microscope and representative images are presented.

2.2.4. Statistical Analysis. All the experiments were carried in quadruples unless otherwise mentioned and the data is represented as mean \pm standard deviation. Data was statistically analyzed using one way analysis of variance (ANOVA) to find the significant difference among different sampling groups. Tukey's test was performed using OriginPro 8.0 software with $* p \leq 0.05$ considered as significant while $*^{*} p \leq 0.01$ as highly significant.

\section{RESULTS AND DISCUSSION}

3.1. Physico-Chemical Studies. There are two unique aspects of the composite bilayered scaffold being reported in this article. The first is the bilayer nature of the scaffold utilizing an underlying osteoinductive sol-gel derived bioactive scaffold, coupled with an upper silk layer to drive regeneration of the cartilage layer. The second aspect was the use of an endemic Indian silk variety, which possesses RGD sequences known to influence cell adhesion and proliferation. ${ }^{18}$ The process parameters such as working distance, voltage and solution parameters were optimized to obtain fluent bead-free fibers.

As can be seen from the scanning electron micrographs (Figure 1), the BM and AA fibers appeared to have smooth surfaces and a circular cross-section. The nanofibers had an average diameter of $175 \pm 53 \mathrm{~nm}$ and $189 \pm 70 \mathrm{~nm}$ for BM and AA mats respectively (Figure 1D). SEM micrographs (Figure $1 \mathrm{~A}$ and $\mathrm{B}$ ) also revealed the porous nature of the $\mathrm{SF}$ electrospun mats. The SF nanofibers were deposited nonuniformly and appeared to intersect each other, forming numerous small pores ranging in size up to several microns. The finely spread nanofibers may serve as a biomimetic template which recapitulates the collagen-II fibrils present in the native cartilage tissue. ${ }^{28}$ Pore size and porosity play a crucial role in this regard. A smaller pore size is desirable for the cartilage phase of the construct, as lower oxygen tension creates a hypoxic environment suitable for maintenance of chondrogenic phenotypes, ${ }^{29}$ and also permits adequate nutrient transfer. The as-spun bioactive glass (BG) mat was also porous, however, the fibers had a larger average diameter of $0.97 \pm 0.34$ $\mu \mathrm{m}$. Importantly, the BG fibers were distributed with an aligned orientation (Figure 1C), similar to the fibrillar pattern of mineralized collagen-I found in the osteonal lamellae. ${ }^{30}$ Interestingly, there was no devoted collector used to attain aligned bioactive glass microfibers. The fibers were oriented in the direction of motion of the rotating mandrel. We hypothesize that a physical drafting effect ${ }^{31}$ could have contributed to this alignment, wherein a state of synchrony between the rotating mandrel speed and the jet stretching speed could have been achieved under the applied parameters for electrospinning. As previously reported, presentation of appropriate microenvironment is crucial for effective cellmaterial interaction,,$^{32-34}$ and larger pores have shown to facilitate faster bone regeneration. ${ }^{35}$ Micrographs of the composite biphasic mat cross sections (Figure $1 \mathrm{E}$ and $\mathrm{F}$ ) demonstrate the significant and successful junction formation of the two different fibrous materials. These micrographs confirm the well-integrated nature of the composite mats and importantly show the maintenance of their porous structure. Additionally, the compact features of upper SF (Figure 1F) were observed showing the small pores (as seen in Figure 1F), and the loosely connected lower BG layer (Figure 1E) due to its microfibrous nature. In earlier reports, microfibrous milieu have shown greater support for growth of osteoblast like cells when compared to nanofibrous environments in vitro, owing to increased porosity associated with the former. ${ }^{36,37}$

Compositional analyses were undertaken using FTIR, XRD and EDX to understand the material's functional properties. FTIR spectra (Figure 2A) were recorded to study the molecular conformation of the silk fibroin and bioactive glass within the electrospun mats (overall FTIR spectra $4000 \mathrm{~cm}^{-1}$ to $500 \mathrm{~cm}^{-1}$ provided in Supporting Information (SI) Figure S1). Characteristic peaks confirming the presence of $70 \mathrm{SiO}_{2} .25 \mathrm{CaO} .5 \mathrm{P}_{2} \mathrm{O}_{5}$

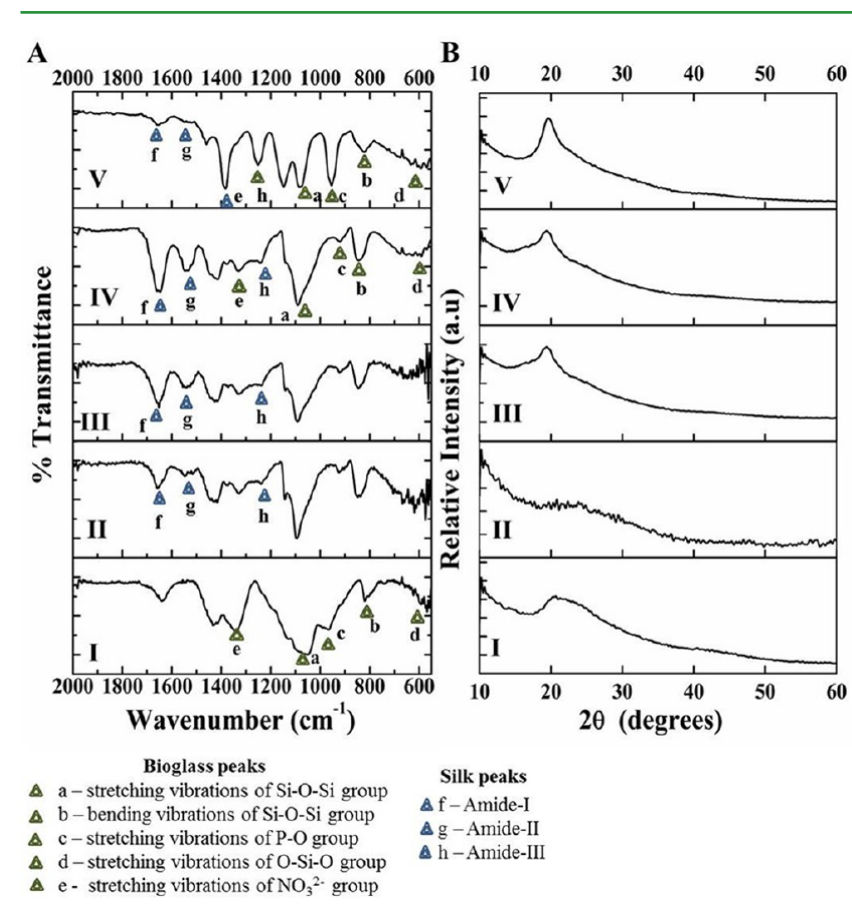

Figure 2. (A) FTIR spectra of electrospun mats and (B) X-ray diffractograms of electrospun mats (I) BG, (II) AA, (III) BM, (IV) BI, and (V) AI mats. 
Table 2. Contact Angle Measurements for Electrospun Mats

\begin{tabular}{|c|c|c|c|c|c|c|c|}
\hline \multirow{4}{*}{$\begin{array}{c}\text { sample } \\
\text { contact angle }\left({ }^{0}\right)\end{array}$} & \multicolumn{3}{|c|}{ monophasic } & \multicolumn{4}{|c|}{ biphasic } \\
\hline & BG & $\mathrm{BM}$ & $\mathrm{AA}$ & \multicolumn{2}{|c|}{$\mathrm{BI}$} & \multicolumn{2}{|c|}{ AI } \\
\hline & $13 \pm 2.6$ & $71.85 \pm 0.8$ & $41.66 \pm 0.6$ & SF Side & $73.51 \pm 1.8$ & SF side & $47.14 \pm 2.9$ \\
\hline & & & & BG Side & $24.2 \pm 2.2$ & BG side & $51.23 \pm 2.2$ \\
\hline
\end{tabular}

A

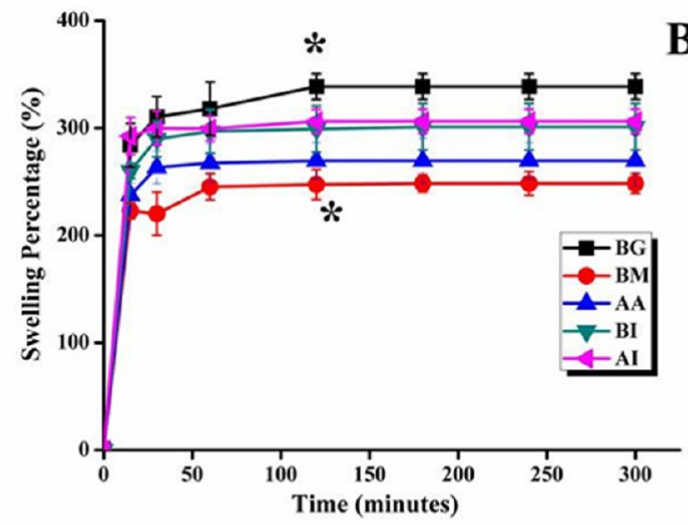

C

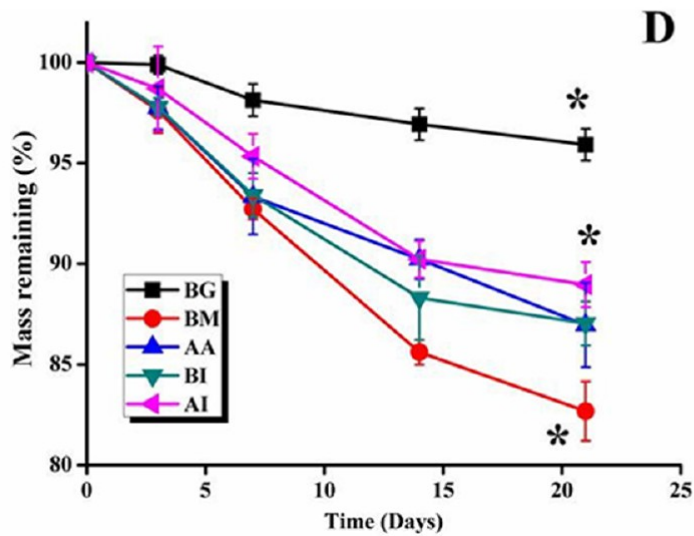

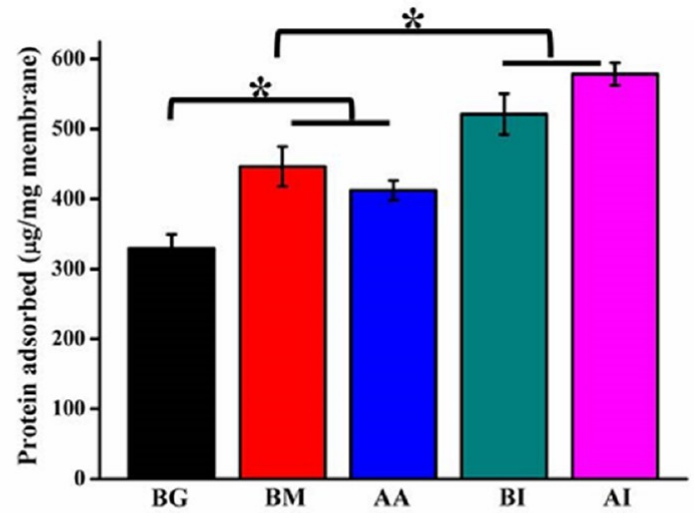

D

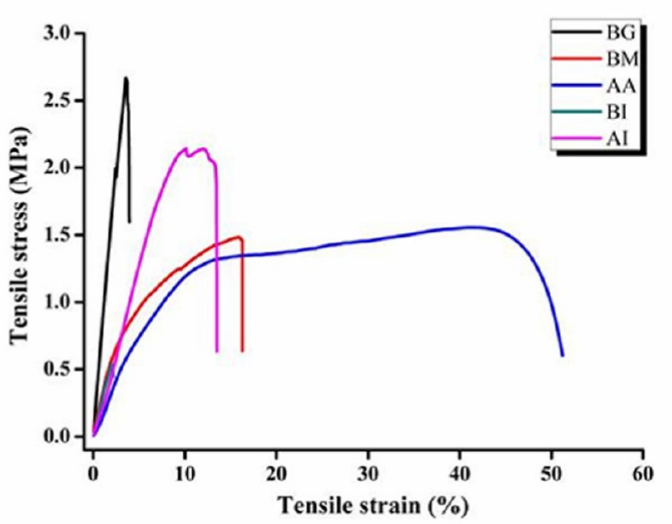

Figure 3. (A) Swelling profile, (B) Protein adsorption profile, and (C) In vitro degradation profile of electrospun mats (* represents statistically significant difference $(p \leq 0.05)$; (D) Representative stress-strain curve for electrospun mats.

bioactive glass were observed at 1042 and $446 \mathrm{~cm}^{-1}$ ( $\mathrm{Si}-\mathrm{O}-\mathrm{Si}$ stretching and bending vibrations), $808 \mathrm{~cm}^{-1}$ (O-Si-O stretching), and $962 \mathrm{~cm}^{-1}$ (P-O stretching), ${ }^{38}$ consistent with compositional analysis obtained from energy dispersive spectra (EDX) (data provided in SI Figure S2). The bone phase present in the basal side of the bilayered construct (BG) would be in direct contact with the subchondral region abundant in bone marrow derived mesenchymal stem cells, therefore the presence of a suitable bioactive ceramic is important to aid differentiation into the osteogenic lineage. The peak at $1378 \mathrm{~cm}^{-1}$ corresponds to a $\mathrm{NO}_{3}{ }^{2-}$ stretching vibration, indicating the presence of residual nitrates in the bioactive glass. ${ }^{39-41}$ In the current study, we resorted in using solvent based stabilization, ${ }^{39}$ wherein we used ethanol to confer insolubilty in silk and to remove nitrates from the bioactive glass and the fabricated composites, rendering the mats suitable for in vitro cell culture studies. The characteristic vibrational regions for silk fibroin were also present in the electrospun mats, as shown in Figure 2A. The amide-I band (corresponding to $\mathrm{N}-\mathrm{H}$ deformation and $\mathrm{C}-\mathrm{H}$ stretching) was seen at $1650-$ $1600 \mathrm{~cm}^{-1}$, amide-II band (corresponding to $\mathrm{C}=\mathrm{N}$ stretching) at $1550-1510 \mathrm{~cm}^{-1}$, and amide-III band was observed at $1260-1210 \mathrm{~cm}^{-1}$ (corresponding to $\mathrm{C}-\mathrm{N}$ stretching). ${ }^{13,23}$ The
$\mathrm{C}-\mathrm{H}$ stretching pertaining to aldehyde, and a broad peak corresponding to the $\mathrm{O}-\mathrm{H}$ group are attributed to the polymers PVA and PVB used for aiding the electrospinning of SF and bioactive glass sol, respectively. ${ }^{42,43}$ It is pertinent to note that both PVB and PVA, used in the current study to adjust the rheological properties of bioactive glass sol and SF, have FDA clearance for use in finished pharmaceuticals as adhesives and components of coatings. ${ }^{44}$ The composite biphasic mats exhibited all the conformational peaks of SF and $\mathrm{BG}$, thus there appeared no alteration in conformation of these materials when spun together. However, the intensity of the amide-I peak at $\sim 1660 \mathrm{~cm}^{-1}$ varied in intensity between the composite materials, suggesting possible differnences in interactions with the $\mathrm{C}=\mathrm{O}$ groups associated with the aldehyde groups of PVB and PVA, ${ }^{45}$ which might have led to the important well integrated interface of the biphasic composites.

X-ray diffraction was employed for further phase analysis of the electrospun mats (Figure $2 \mathrm{~B}$ ). The broad or weak peaks observed at $\sim 20^{\circ}$ correspond to the $\beta$-sheet, indicating that the $\mathrm{SF}$ is amorphous in nature within the electrospun silk matrices. $^{46-48}$ The amorphous nature of the SF mats may be attributed to the rapid evaporation of the solvent, slow rate of 
Table 3. Tensile Properties for Electrospun Mats

\begin{tabular}{|c|c|c|c|c|c|}
\hline & BG & $\mathrm{BM}$ & AA & BI & AI \\
\hline elastic modulus ( $\mathrm{MPa})$ & $86.66 \pm 4.32^{a}$ & $13.42 \pm 0.16^{b}$ & $22.90 \pm 1.76^{c}$ & $29.36 \pm 1.38$ & $27.48 \pm 3.96$ \\
\hline elongation at break (\%) & $3.83 \pm 0.33^{a}$ & $15.98 \pm 0.89^{b}$ & $55.11 \pm 12.84^{c}$ & $2.28 \pm 0.59$ & $8.52 \pm 1.43$ \\
\hline
\end{tabular}

${ }^{a}$ Represents statistical significance between BG and rest of the group at $p \leq 0.05$. ${ }^{b}$ Represents statistical significance between BM and BG, AA at $p \leq$ 0.05 . ${ }^{c}$ Represents statistical significance between $\mathrm{AA}$ and $\mathrm{BM}$ at $p \leq 0.05$.

crystallization, and the short travel time of the jet in air using electrohydrodynamic atomization. ${ }^{49}$ Furthermore, the XRD pattern obtained for the $70 \mathrm{~S}$ bioactive glass (BG) mat (Figure 2B I) confirmed the amorphous nature of the bioactive glass, as expected for a sol-gel bioactive glass samples without thermal treatment. Sol-gel derived bioactive glasses remain advantageous over the conventionally available melt derived bioactive glasses, due to their high surface area to volume ratio and faster resorption rate in vivo. ${ }^{50}$ In addition, the dissolution products of amorphous bioactive glasses, owing to their faster dissolution rate, can stimulate proliferation and differentiation of bone marrow derived stem cells. ${ }^{51}$ This was evident in the biological studies as reported in subsequent sections, thereby validating the functionality of the fabricated mats.

Hydrophobicity and/or hydrophilicity of electrospun materials are critical parameters and can be one of the main controlling factors determining the events at the cell-matrix interface. These parameters were assessed by measuring the water contact angles (Table 2). Measurements indicated that AA mats were more hydrophilic than BM mats and this had important implications for subsequent biological measurements. Nonmulberry silk varieties belonging to the Saturniidae family (A. assama) are abundant in poly alanine repeats, whereas the silk belonging to the Bombycidae family contain poly glycine-alanine repeats. These poly alanine repeats confer more $B$-sheet formation in non-mulberry than in mulberry silk. ${ }^{18,52}$ The poly alanine and the poly glycine-alanine repeats dictate the self-assembly process of the regenerated silk fibroin solution into final conformation in scaffolds. Hence, the mulberry silk (BM) possesses a more hydrophobic surface when compared to the non-mulberry (AA) silk; where in the latter all the hydrophobic regions are very well embedded within the core with only hydrophilic regions exposed.

There was an increase in contact angle observed upon combining SF with BG fibers for both composite mats (BI and $\mathrm{AI}$ ); this increase was largest for the $\mathrm{BG}$ side of the combined mats, showing an increase from $13^{\circ}$ for BG alone to $24^{\circ}$ and $51^{\circ}$ for $\mathrm{BI}$ and $\mathrm{AI}$ mats, respectively. During the electrospinning process, the biopolymer jet discharged from the needle rapidly evaporates and deposits polymer over the collecting mandrel. The resulting supramolecular assembly ensures the interaction of poly alanine and poly glycine-alanine repeats and subsequent condensation and molecular rearrangement into $B$-sheets. The amino acid compositional variability between the two silks may have led to the differences in interaction with the spun primary bioactive glass layer. Furthermore, the porosity and relatively small depth (ca. $1-2 \mu \mathrm{M}$ ) of the bioactive glass layer when in the composite material would reduce its hydrophilicity in comparison to the bulk material. This may explain the difference in the contact angles, a crucial factor for cell adhesion and growth. Importantly, the varying water contact angles of the different sides of composite mats (BI in particular) demonstrated that this material could provide both hydrophilic and hydrophobic surface functionality.
The extent of wettability possessed by a biomaterial is related to its surface property. The scaffolding material when placed inside the body must be able to absorb the body fluid, thus supporting the nutrient and metabolite transfer between the scaffolding construct and surrounding tissue in contact. The swelling profile of the electrospun mats is shown in Figure 3A. All the mats attained their maximum swelling capacity within 2 $\mathrm{h}$, with the BG mat exhibiting the highest swelling percentage of around $330 \%$, while the silk mats recorded swelling percentages around $250 \%$, statistically significant with respect to the $\mathrm{BG}$ mats $(p \leq 0.05)$. The composite mats exhibited swelling percentage of $300 \%$, with no significant difference noticed between the composite mats. The increased swelling percentage in BG mats may be attributed due to its hydrophilic nature in comparison to silk mats, which may play key roles in cell adhesion and extracellar matrix protein deposition. Though there was difference in contact angle noticed between the silk mats, there was no significant difference between the groups. This may be attributed to the hydrophilic nature of PVA within the silk matrix, which might have enhanced the water retention capacity. Similarly, the swelling percentage of the composite mats ranged between that of the hydrophilic BG mats and the silk mats. The surface of the implant also mediates the adsorption of proteins when it comes in contact with physiological fluids. These adsorped proteins further regulate the cell-matrix interaction. Bovine serum albumin (BSA), having a close likeliness to human serum proteins, ${ }^{24}$ was chosen as a model protein to study the adsorption profile (given in Figure $3 \mathrm{~B}$ ) of the developed electrospun mats. It was noticed the silk mats exhibited higher protein adsorption, ca. 1.3 folds higher $(p \leq 0.05)$ than the BG mats. The composite mats exhibited the highest protein adsorption, ca. 1.2 folds higher than silk mats and ca. 1.7 folds higher than BG mats. The adsortpion was possibly mediated via electrostatic or van der Waals interaction, proving the composite mats' potency as biologically recognizable materials.

The rate of scaffold degradation plays an important role during the regeneration process when implanted in vivo. The degradation process is assisted synergistically under in vivo conditions by various ECM modulatory enzymes, such as matrix metalloproteinases. ${ }^{53}$ In order to achieve the same functional performance offered by these enzymes, a nonspecific proteolytic enzyme, protease XIV was chosen to carry out the degradation studies in vitro. ${ }^{23}$ All the mats showed a time dependent mass loss as observed from Figure 3C. Among the silk mats, BM exhibited a faster rate of degradation in comparison to AA. The AA retained about $87 \%$ mass, whereas $\mathrm{BM}$ retained about $82 \%$. The slower degradation rate of $\mathrm{AA}$ may be attributed to the strong hydrophobic interactions of polyalainine repeats found in non-mulberry silk, rendering protease XIV inaccessible for proteolytic cleavage. The BG mats, however, retained ca. $94 \%$ ( $p \leq 0.05$ in comparison to silk mats) of their mass after 21 days as there was no protein component associated with it. The leaching out of inorganics may be a plausible reason for the observed mass loss. Between 
the composite mats there was no significant difference noticed and they retain $87 \%$ of the mass after 21 days.

Tensile strength investigations provided insightful inputs about the mechanical properties of the electrospun mats. Stress-strain curves representing the different electrospun mats used in this study are shown in Figure 3D, with the data summarized in Table 3. The BG mats exhibited the highest elastic modulus of $86 \mathrm{MPa}$ and the lowest strain percentage of ca. $3.8 \%$, characteristic of a ceramic doped polymer. In comparison, the silk mats $\mathrm{BM}$ and AA both exhibited higher strain percentage before fracture, further evidencing the ductile properties possessed by the SF biopolymer. AA exhibited significantly higher elastic modulus $(23 \mathrm{MPa})$ than $\mathrm{BM}$, and a higher breaking strain (55\% compared to $16 \%$ for BM). This may be due to the poly alanine repeats present in the nonmulberry silk varieties, which impart superior mechanical properties for cartilage repair compared with mulberry silk (containing poly glycine-alanine repeats). ${ }^{54}$ There was no significant difference between elastic modulus of composite mats, both recording about 27-29 MPa. In the composite mats, the cumulative increase in the initial elastic region (Figure 3D) could be attributed jointly due to silk and bioactive glass. This was followed by plastic deformation, which was relatively shorter in the case of BI mats, whereas the AI mats exhibited a longer plastic deformation region, consistent with the larger breaking strain exhibited by AA alone. The plastic deformation is followed by a nonlinear plateau region indicating delamination of the construct and eventually failure. Thus, the composite mats, especially AI, demonstrated similar biomechanics to that seen in an osteochondral interface (consisting of the elastic hyaline cartilage), which constantly deforms under articular movement and the subchondral bone which resists the compressive force. Therefore, the silk phase should well approximate the mechanical properties for the cartilage region and the bioactive glass to bone.

3.2. Biological Studies. 3.2.1. Cell Proliferation and Imaging. In vitro biological studies were carried out to investigate the differential behavior of the seeded osteoblasts and chondrocytes on the electrospun matrices. The utilization of a two cell-type culture model for the composite biphasic mats is complex, but important due to the interaction that occurs between the cell types. All the tested mats supported the growth of cells seeded on them, exhibiting no discernible cytotoxicity. Cell proliferation was assessed using the alamar blue assay (shown in Figure 4), the chondrocytes and osteoblasts were cultured on BG, BM, AA mats separately as controls and cocultured on the composite mats (BI and $\mathrm{AI}$ ) for 14 days. Both the silk mats (AA and BM) aided the proliferation of the chondrocytes better than their $B G$ counterpart (with ca. 1.2 fold increase with respect to BG). However, it was observed that the AA mats supported the greater proliferation of both chondrocytes and osteoblast cells, in particular displaying a 1.3 fold and 1.5 fold increase compared to $\mathrm{BG}$ and $\mathrm{BM}$ respectively for osteoblast cells. $A$. assama possesses intrinsic tripeptides-RGD (arginine-glycineaspartate) toward the $\mathrm{N}$ and $\mathrm{C}$ terminals of glycine rich Gmotif. This particular tripeptide has been widely shown to enhance cell attachment via integrin receptor mediated pathway. ${ }^{55}$ Therefore, it can be hypothesized that the greater cell proliferation supported by the AA mats may be due to the presence of these intrinsic RGD peptides, which mediated the cell attachment and subsequently regulated the cell proliferation. ${ }^{56}$ Importantly, the composite mats supported the growth

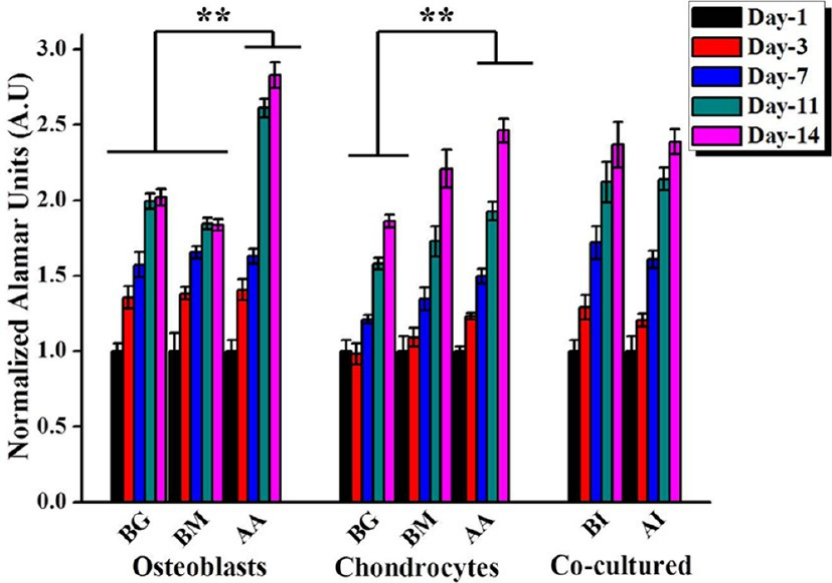

Figure 4. In vitro cell proliferation assessment using alamar blue cell viability assay on electrospun mats $(* *$ represents statically significant difference $p \leq 0.01$ ).

of both the cell types in the 14 day culture, and no significant difference was seen between the composite mats.

The suitability of electrospun mats as tissue engineering constructs were further evaluated using live/dead staining (Figure 5A). It was observed that the non-mulberry silk mat

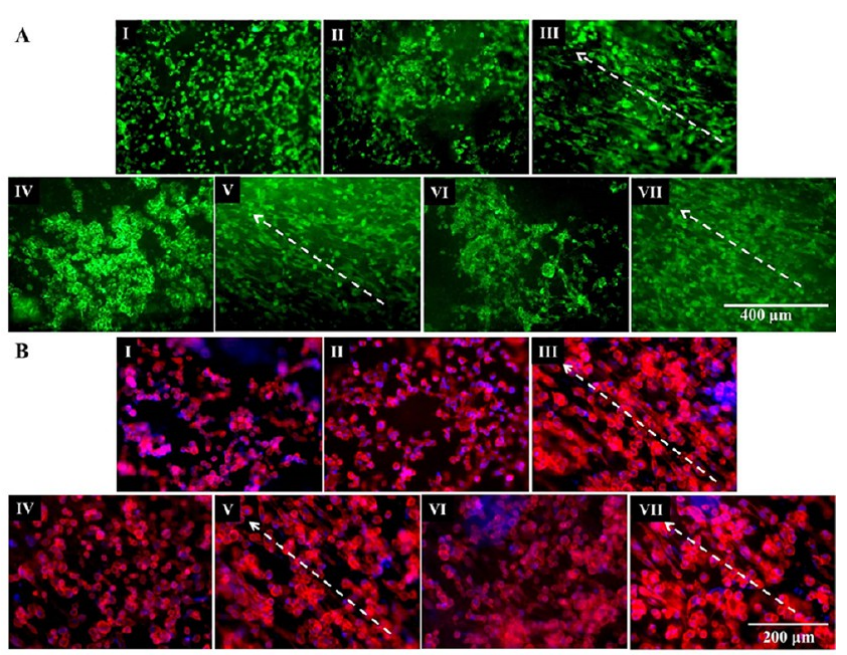

Figure 5. (A) Live/Dead imaging and (B) cytoskeletal architecture assessment of seeded cells; chondrocytes grown on (I) BM and (II) AA mats, osteoblasts grown on (III) BG mats, chondrocytes grown on silk side of (IV) BI, (VI) AI composite mats and osteoblasts grown on bioactive glass side of (V) BI and (VII) AI composite mats (white dashed arrows indicating direction of alignment of cells along the fibrillar direction).

(AA) showed higher live cell density compared to its mulberry counterpart, correlating with findings from the cell proliferation testing. The coculture system on the composite mats supported the growth of both cell types, with no adverse effects over the full 14 days, in keeping with the alamar blue assay results (Figure 4). Furthermore, some orientation of the cell growth can be seen in these images, driven by the oriented fibrillar nature of the scaffolds. This was also evident in the cytoskeletal image measurements detailed below.

In order to evaluate the cell cytoskeletal architecture, actin filaments were stained using rhodamine conjugated to phalloidin (Figure 5B). The osteoblasts seeded on BG mats 

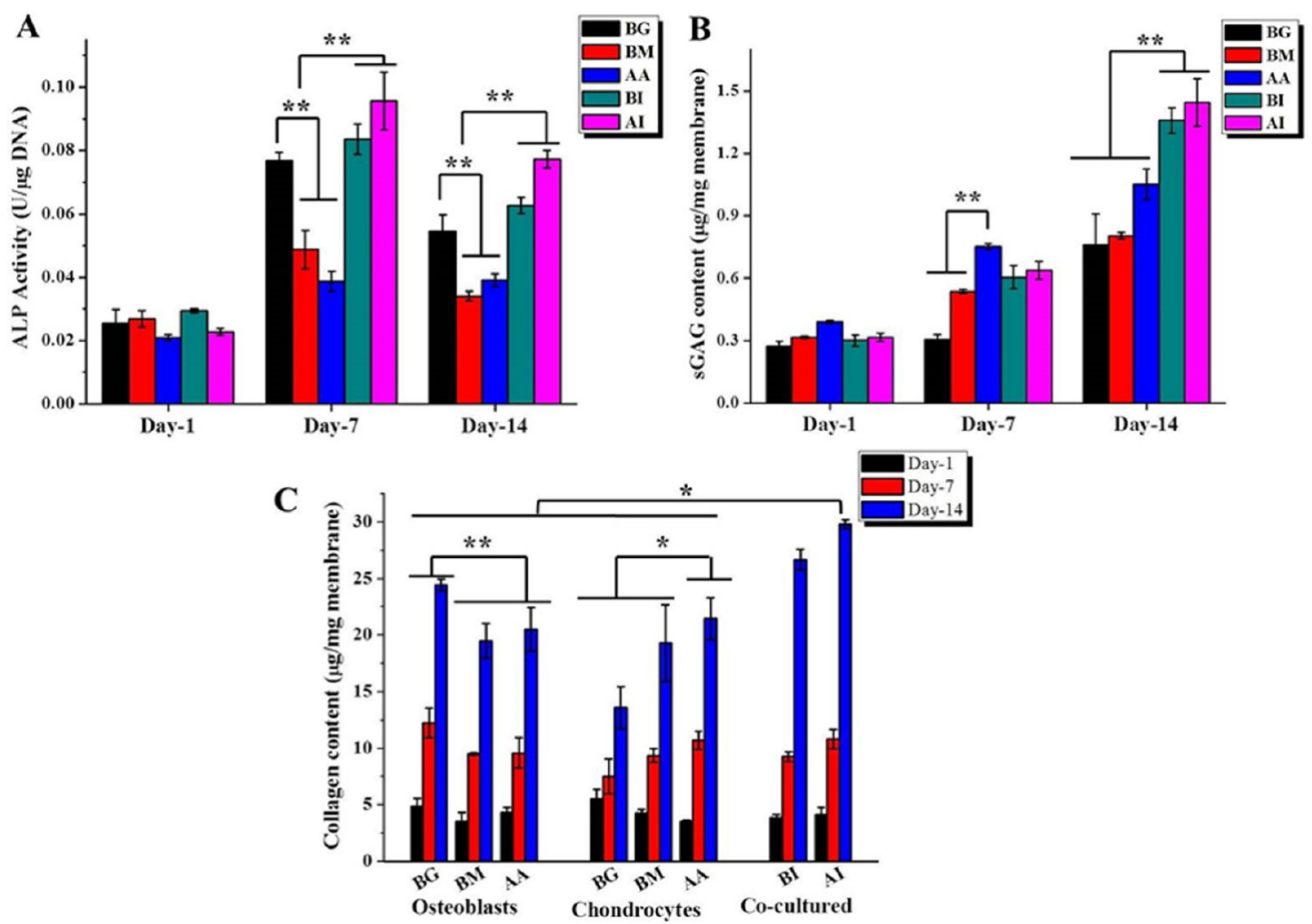

Figure 6. Biochemical assessment of cell seeded mats; (A) alkaline phosphatase activity estimation, (B) sulfated glycosaminoglycan estimation, and (C) total collagen estimation (* represents significance level at $p \leq 0.05$ and ** represents significance level at $p \leq 0.01$ ).

aligned themselves along the direction of the nanofibers and spread along the fibril axis. Clusters of rounded morphology cells were observed alongside the oriented cells after 14 days, which appear to be bone-nodule like cells (as seen in Figure 5B III, V, VII) formed from initially adherent cells. The aligned fibrillar organization is similar to the native osteonal lamellae in the cortical bone, which might have led to this as mentioned secondary clustered pattern.

The chondrocytes grown on plastic (Tissue culture plasticTCP, shown in SI Figure S3) lost their phenotype and acquired a fibroblastic phenotype, characterized by spreading-out of cells and reduction in synthesis of cartilage specific proteins. ${ }^{58}$ In comparison, the chondrocytes grown on silk mats acquired a rounded morphology pertaining more to their native morphology. Furthermore, chondrocytes cultured on individual and composite AA mats were in clusters and well distributed throughout. This may be attributed to the hydrophilic nature of the AA mats in comparison to BM mats which exhibited a higher water contact angle.

3.2.2. Biochemical Analysis and Gene Expression Profiling.

To assess the progression and state of chondrogenesis and osteogenesis, biochemical analysis of different marker proteins and gene expression profiling of marker genes were performed. Chondrogenesis is marked by precursor cells undergoing condensation into clusters, followed by their proliferation and expression of ECM proteins. Among the ECM proteins collagen-II, glysoaminoglycan and aggrecan are the principal proteins expressed in abundance that help in forming the cartilaginous matrix. $^{6,59}$ The hallmark feature of osteogenesis is the expression of collagen-I protein by osteoblasts on to which the calcium nucleates, and apatite crystals form. This results in a mineralized matrix followed by the expression of modulatory proteins like osteocalcin, osteopontin and BSP which regulate the crystal growth. ${ }^{60}$ Alkaline phosphatase (ALP), a key regulatory enzyme in the mineralization process was used as a marker to check the osteoconductive potential of the mats (Figure 6A). The level of ALP expression peaked after 7 days, with BG mats showing significantly higher levels than osteoblast seeded silk only mats (ca. 1.6 fold higher than $\mathrm{BM}$, whereas ca. 2.02 fold higher than $\mathrm{AA}$ ), which then gradually decreased, indicating the onset of mineralization. This is concurrent with the use of amorphous $70 \mathrm{~S}$ bioactive glass which is a potent regulator of osteogenesis ${ }^{61}$ and whose ionic products mediate the differentiation of osteoblasts. The ALP levels reduced at day 14, which may be an indication of terminal maturation of the osteocytes. On the composite mats, where the chondrocytes and osteoblasts were cocultured, there was a significant increase in the ALP expression (with BI showing ca. 1.1 fold increase and AI showing ca. 1.3 fold increase in comparison to BG mats). This increase in ALP activity may be explained as the combined action of osteoblast and chondrocytes in coculture, which has been reported previously both in micromass culture ${ }^{62}$ as well as in scaffold systems ${ }^{63}$ to enhance mineralization. Furthermore, the AI mats showed significant increase $(p \leq 0.01)$ in ALP expression compared to the $\mathrm{BI}$ composite mats, further demonstrating the excellent osteoconductive potential of the composite mats containing A. assama silk.

Sulfated glycosaminoglycans (sGAG), one of the main extracellular matrix components secreted by chondrocytes were quantified within the scaffolds to evaluate their chondrogenic potential (Figure 6B). The sGAG content varied significantly between the mats, with the silk mats (AA and $B M$ ) exhibiting higher levels in comparison to BG (at day 14, AA 
A

I

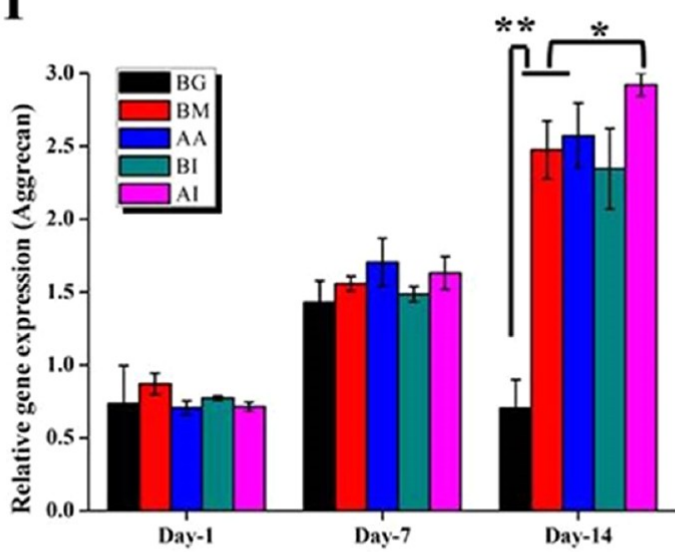

B

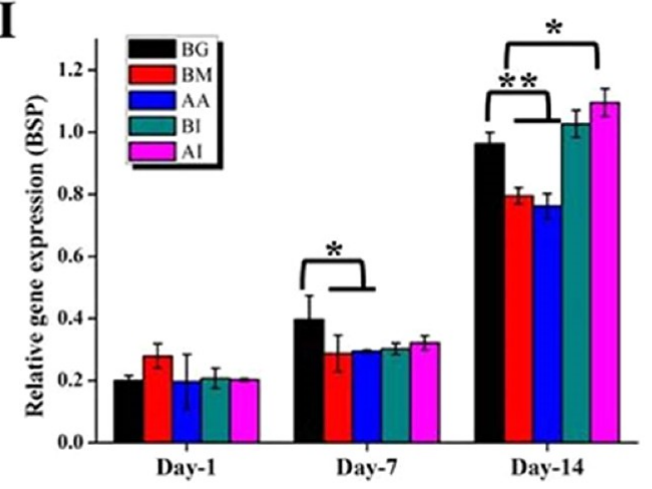

II
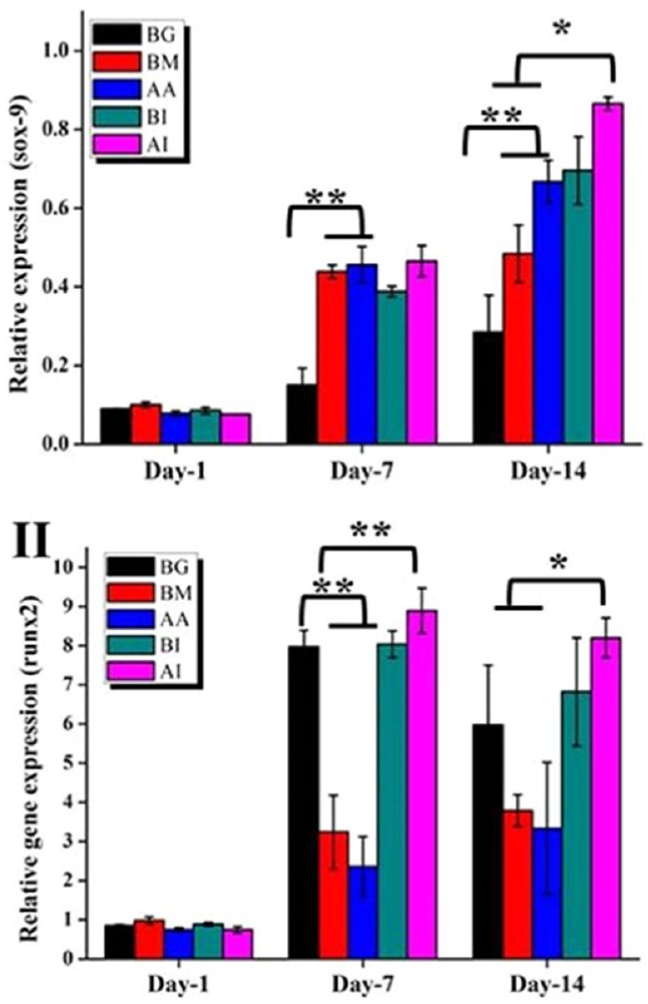

Figure 7. Real time gene expression profile for (A) chondrogenic genes (I) aggrecan and (II) sox-9; for (B) osteogenic genes (I) BSP and (II) runx2. (* represents significance level at $p \leq 0.05$ and ** represents significance level at $p \leq 0.01$ ).

exhibited ca. 1.5 fold increase and BM exhibited ca. 1.1 fold increase). The composite mats where the cells were cocultured also showed the greatest increase in sGAG after 14 days, in line with results seen from the ALP studies. Similarly, the AI mats exhibited the highest sGAG content (at day 14, ca. 1.4 fold increase in comparison to AA). This may be attributed to the hydrophilic surface provided by the AA mat. Ma et al., studied the effect of chondrogenesis on hydrophobic PLLA surface and PLLA surface modified by addition of hydrophilic groups, ${ }^{64}$ it was observed that modified hydrophilic PLLA surfaces exhibited better cell adhesion and matrix protein deposition. ${ }^{65}$ In addition, chondrocytes express several integrin receptor families which help to maintain their homeostasis. Due to the AA mats' abundance in RGD tripeptide, it may be hypothesized that integrin binding stimulates intracellular signaling and subsequent ECM production. ${ }^{66}$

In order to evaluate the extent of ECM deposition on the electrospun mats, total collagen content was quantified (Figure $6 \mathrm{C})$. It was observed that osteoblasts seeded on BG secreted higher collagen (ca. 1.25 fold higher than BM and ca. 1.19 fold higher than AA) than silk mats. Conversely, chondrocyte seeded AA mats exhibited higher collagen (ca. 1.57 fold increase in comparison to BG) content when compared to BG and BM mats. Among the osteoblast seeded mats BG exhibited higher collagen deposition, correlating with measured ALP activity (Figure 6A). The terminal maturation is characterized by an increase in mineralized matrix which is correlated with an increased collagen deposition. Collagen content was highest in the composite mats where the osteoblasts and chondrocytes were cocultured, concurrent with sGAG and ALP results. Furthermore, after 14 days AI mats exhibited a ca. 1.12 fold increase in comparison to BI bilayered composite mats, correlating with the excellent osetoconductivity and retainment of chondrogenic phenotype observed for the non-mulberry silk composite mats during sGAG and ALP quantification.

Chondrogenic marker genes aggrecan and sox-9, and osteogenic marker genes bone BSP and runx 2 were analyzed to further evaluate the chondrogenic and osteogenic potential of the cell seeded constructs over a period of 14 days. Greater up-regulation of cartilage specific genes aggrecan and sox-9 (as seen in Figure 7A I and II) was observed in chondrocyte seeded silk mats, in particular AA, further demonstrating the better suitability of these materials for chondrocyte cultivation (ca. 2.35 fold increase in sox- 9 expression and ca. 3.67 fold increase in aggrecan, in comparison to BG). Concurrent with ALP analysis results, up-regulation of bone specific gene BSP (Figure $7 \mathrm{~B} \mathrm{I})$ was found to be higher in osteoblast seeded BG mats in comparison to silk (BM and $\mathrm{AA}$ ) mats, confirming the excellent potential for osteoblast cultivation. In the case of runx2, the expression for BG was ca. 2.4 folds higher than BM and ca. 3.4 fold higher than AA. Runx2 an early differentiation marker expressed by committed osteoblast cells, marks the onset of mineralization process. High expression levels of runx2 (Figure $7 \mathrm{~B}$ II) at day 7 in osteoblast seeded BG mats and cocultured composite mats attested to the fact that the osteoblasts were moving toward acquiring osteocyte like phenotype and terminal maturation, in comparison to the osteoblasts seeded on the silk mats. Higher expression levels of BSP (at day 14), a 
noncollagenous protein which helps in nucleation and modulating the apatite crystal growth ${ }^{67}$ concurred with the runx2 experiment findings. Importantly, it was found that sox-9, a key early chondrogenic stage marker had a higher expression in the composite (AI) mats affirming the commitment of chondrocytes toward the chondrogenic lineage. ${ }^{10}$ Likewise, expression of aggrecan, an important proteoglycan constituent of ECM is involved in recruitment of sGAG and chondroitin sulfate during the condensation of chondrocytes to form functional clusters which later lay the template for the cartilaginous matrix. ${ }^{12}$ The increased level of expression of aggrecan (involved in recruitment of sGAGs and chondroitin sulfate during the condensation of chondrocytes to form functional clusters which later lay the template for the cartilaginous matrix ${ }^{12}$ ), in line with the higher levels of sGAG recorded in the composite mats (in particular AI). These results attest the maintenance of chondrogeneic phenotype of the seeded chondrocytes, which was marked by the clustering of cells (Figure 5A VI and B VI). This observation was also in accordance with the results observed for collagen deposition, reaffirming the significant potential of these composite scaffolds for OCD treatment.

3.2.3. Histological Assessment and Immunostaining of Marker Proteins. Due to the nature of both the scaffolds and the osteogenic-chondrogenic coculture model, these samples readily lend themselves to histological sectioning. To assess cell attachment and distribution, transverse sections were stained with hematoxylin and eosin (Figure 8), revealing that the cells were present on the surface of all the mats, with little infiltration.

Microfibrous scaffolds have been shown to support the infiltration and survival of osteoblasts when compared to nanofibrous matrices. ${ }^{37}$ The microfibrous nature of the bioactive glass as discussed earlier, allows for bigger pores than the nanofibrous SF. This led to better infiltration of cells in the bioactive glass mats (Figures $8 \mathrm{~A}, \mathrm{~F}$, and $\mathrm{H}$ ) in comparison to SF mats. Sections of chondrocytes cultured on AA mats (Figure 8C) revealed a compact structure and a well adherent multilayer owing to its better surface properties, whereas BM mats (Figure 8B) showed a loose adhesion profile. The composites mats supported the growth of both cell types as seen in Figure $8 \mathrm{D}$ and $\mathrm{E}$. The chondrocytes cultured on AI (Figure 8I) exhibited a compact multilayer, while the chondrocytes grown on BI mats' silk side (Figure 8G) were loosely bound in a disrupted multilayer.

In order to further evaluate the ECM deposition on the electrospun matrices the sections were stained with alcian blue and alizarin red to differentially stain sGAG secreted by chondrocytes, and calcium deposits by osteoblasts respectively (Figure 9A I to VI and 9B I to VI). The BG mats showed mineral deposits which were stained red (Figure 9A I), and appeared well infiltrated (Figure 9A IV) within the matrix. The AA mats revealed dense alcian blue accumulation over the compact multilayer cell stacks (Figure 9A III), with clusters indicative of chondrocyte aggregates (shown in Figure 9A VI), resulting from higher aggregan expression (backed by the gene expression results). Conversely, the cells were loosely bound on the BM mats (Figure 9A II and V). Both the cell types were supported on the composite mats as indicated by the differential stain uptake, the bioactive glass side taking up an intense red coloration and the SF side taking up a dense blue coloration (Figure 9B I and II). Moreover, the distinguishable presence of marker proteins OPN for bone matrix and

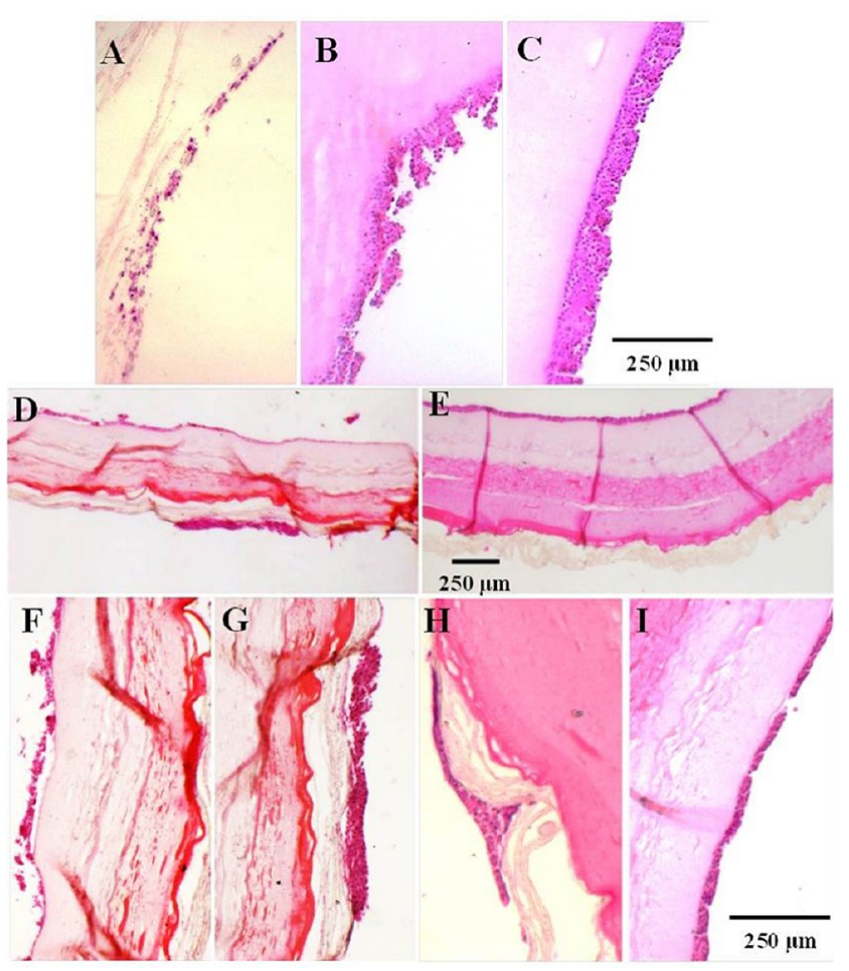

Figure 8. Histological assessment-Hematoxylin and eosin stained sections; (A) BG mats seeded with osteoblasts, (B) AA, and (C) BM mats seeded with chondrocytes; (D) AI and E) BI composite mats with chondrocytes and osteoblasts are cocultured, whereas $(\mathrm{F})$ and $(\mathrm{H})$ are bioactive glass side with osteoblasts grown on it, (G) and (I) are silk side with chondrocytes grown on it, of BI and AI composite mats respectively (dashed arrow showing infiltration of osteoblasts, arrow representing condensed chondrocytes on the silk matrix).

collagen-II for cartilage matrix in the electrospun mats were probed using immunostaining (Figure 9A VII to IX and 9B VII to $\mathrm{X}$ ). OPN, one of the main noncollagenous proteins involved in apatite crystal modulation during mineralization process, serves as mid-late stage osteogenic marker. ${ }^{67}$ The BG mats showed higher expression of OPN (Figure 9A VII), in unanimity with increased ALP, runx2 and BSP expression, reiterating the BG mats' osteoconductive nature. The SF mats all showed expression of collagen-II (Figure 9A VIII and IX), with the AA mats exhibited relatively higher levels of collagen-II in comparison to BM. Similarly, expression of collagen-II was shown in the composite mats. Collagen-II is an important ECM protein responsible in giving the cartilaginous matrix the mechanical resilience needed during mechanical stress resistance. $^{6}$ It is important to note that collagen-II expression correlated with the results observed in collagen estimation (Figure 6C), proving the composite mats' ability to sustain matrix biosynthesis.

3.2.4. In Vitro Immune Response Assessment. Any adverse immune reactions elicited by a biomaterial would result in acute outcomes such as inflammation, tissue destruction, as well as interference in the healing process resulting in rejection of the graft. ${ }^{68}$ Therefore, as a quantifiable precursor to in vivo testing, the in vitro immunogenicity of the mats were tested using murine macrophage cells at 12 and $24 \mathrm{~h}$, using the TNF- $\alpha$ secretion. It can be seen from Figure 10, that all the mats exhibited negligible immune response comparable to negative control, demonstrating that the mats would not induce any adverse immune response. Silk based biomaterials which have 


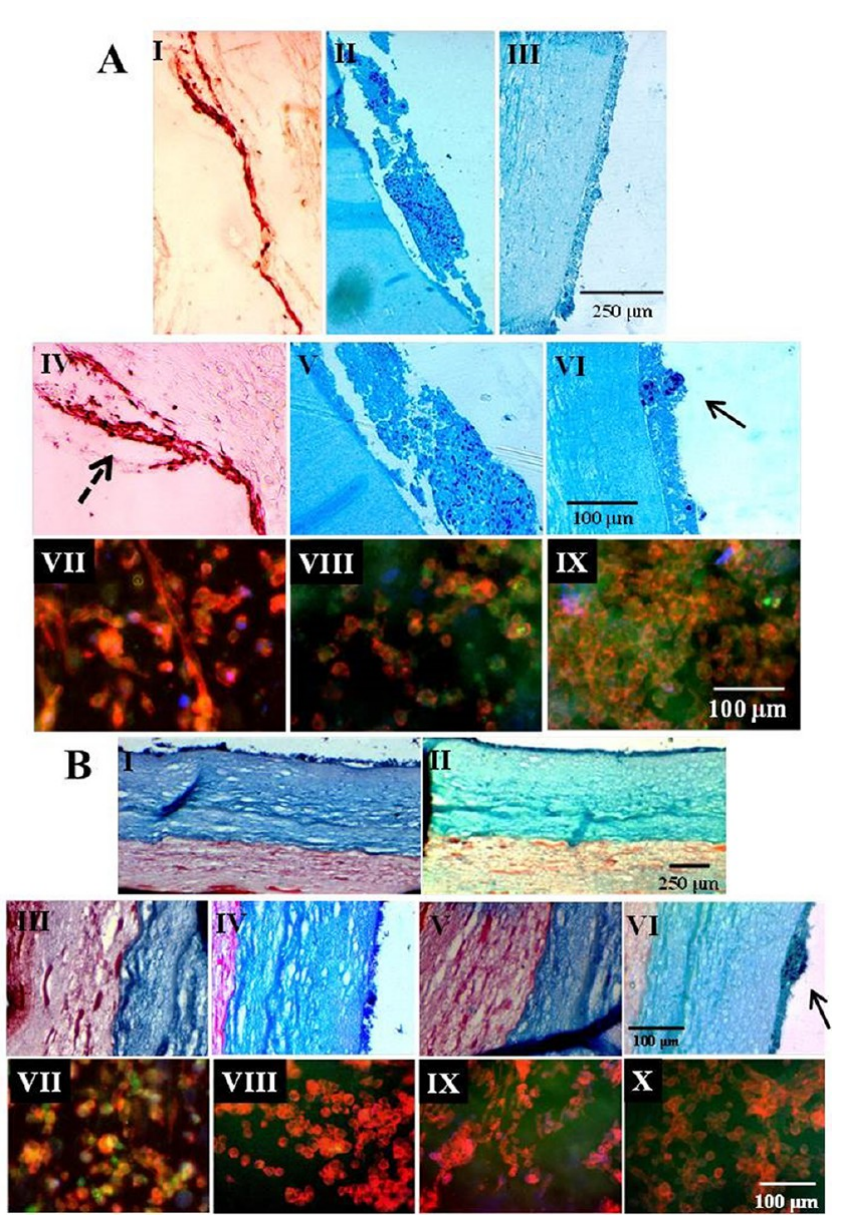

Figure 9. Histological assessment-alizarin red and alcian blue stained transverse sections of (A) control group consisting of (I) osteoblast seeded BG mats, (II), and (III) chondrocyte seeded BM and AA mats respectively; (B) experimental group consisting of (I) BI and (II) AI composites mats conducive for coculture of (III), (V) osteoblast seeded bioactive glass side retaining alizarin red, and (IV), (VI) chondrocyte seeded silk side retaining alcian blue. Expression of marker proteins-osteoblasts were stained for OPN in (A.VII) BG, (B.VII) BI, and (B.IX) AI mats; chondrocytes were stained for collagen-II in (A.VIII) BM, (A.IX) AA, (B.VIII) BI, and (B.X) AI mats respectively (dashed arrow showing infiltration of osteoblasts, arrow representing condensed chondrocytes on the silk matrix).

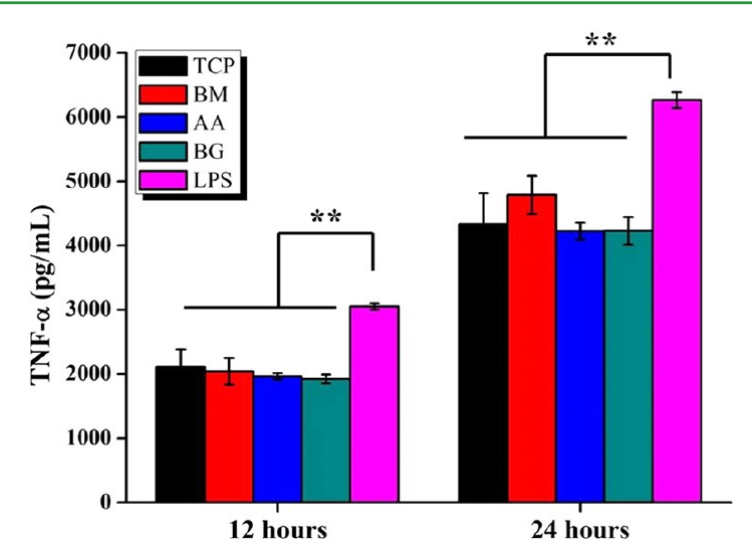

Figure 10. In vitro immune response assessment of electrospun mats by measuring TNF- $\alpha$ release from murine macrophage RAW 264.7 cells $(n=2, * *$ represents significant difference at $p \leq 0.01)$. been shown to be immunocompatible in vitro were found to elicit negligible immune response in vivo. ${ }^{23,69}$ Therefore, these materials are expected to be applicable for successful implementation in vivo without further processing.

\section{CONCLUSIONS}

In the current study, we have reported a facile, scalable, and reproducible strategy for the development of electrospun bilayered composite mats for osteochondral defect repair. We utilized a layer by layer approach wherein the bioactive $70 \mathrm{~S}$ bioactive glass sol was electrospun as the first layer followed by the silk layer. The mats exhibited a coherent, well integrated interface having two distinct phases to individually support the growth and maturation of osteogenic and chondrogenic cells. The biphasic structure was shown to provide a spatially confined biomimetic micromilieu similar to the osteochondral interface. A systematic study of physical, mechanical, and biological characteristics of the electrospun composite mats revealed that non-mulberry silk based AI mats performed better in comparison to the mulberry silk based BI mats. The former not only exhibited better tensile properties mimicking the biomechanics encountered at the osteochondral interface, but also showed superior cell supportive characteristics. Furthermore, biochemical studies indicated enhanced ALP activity, sGAG and collagen secretion with these materials suggesting phenotypic maintenance; which was corroborated by the expression of OPN and collagen-II observed in immunostaining of the seeded osteoblasts and chondrocytes, and expression profiling of bone and cartilage associated genes in cell seeded composite mats. However, maturation of the cell seeded construct under dynamic culture condition is needed to circumvent the cell infiltration limitations. In vitro experiments demonstrated that the composite materials should not induce any adverse immune response; therefore further validation of these materials in vivo is of great interest, which will form the basis of a future study. In conclusion, the developed novel BG/ SF composites possess great promise for osteochondral graft materials amenable for OCD repair and management.

\section{ASSOCIATED CONTENT}

S Supporting Information

The Supporting Information is available free of charge on the ACS Publications website at DOI: 10.1021/acsami.6b16590.

FTIR spectra of electrospun mats; EDX spectra for BG mats; Fluorescent images of chondrocytes and MG63 cells (PDF)

\section{AUTHOR INFORMATION}

\section{Corresponding Authors}

*(J.C.K.) Phone: +44-(0)20-7915-1189; fax: +44-(0)20-79151227; e-mail: j.knowles@ucl.ac.uk.

*(B.B.M.) Phone: +91-361-258-2225; fax: +91-361-258-2249; e-mail: biman.mandal@iitg.ernet.in.

\section{ORCID}

Biman B. Mandal: 0000-0003-3936-4621

\section{Notes}

The authors declare no competing financial interest.

\section{ACKNOWLEDGMENTS}

B.B.M. and J.C.K. thankfully acknowledge the generous funding aided through Department of Science and Technology - UK- 
India Education and Research Initiative project (UKIERI Grant No. DST/INT/UK/P-110/2014). BBM thankfully acknowledges the funding support through the Department of Biotechnology (DBT, Grant nos. BT/PR6889/GBD/27/490/ 2012 and BT/548/NE/U-Excel/2014) and the Department of Science and Technology (DST, Grant no. SB/FT/LS-213/ 2012). J.C.M. acknowledges the Ministry of Human Resource Development (MHRD) for his fellowship. RK is thankful to IIT Guwahati for the receipt of his institutional postdoctoral fellowship. PJTR acknowledges the financial support of the EPSRC (Grant no. EP/L026287/1).

\section{REFERENCES}

(1) Kurtz, S.; Ong, K.; Lau, E.; Mowat, F.; Halpern, M. Projections of Primary and Revision Hip and Knee Arthroplasty in the United States from 2005 to 2030. J. Bone Joint Surg. 2007, 89 (4), 780-785.

(2) Pachore, J. A.; Vaidya, S. V.; Thakkar, C. J.; Bhalodia, H. K. P.; Wakankar, H. M. ISHKS Joint Registry: A Preliminary Report. Indian J. Orthop. 2013, 47 (5), 505-509.

(3) Getgood, A.; Bhullar, T.; Rushton, N. Current Concepts in Articular Cartilage Repair. Orthop. Trauma 2009, 23 (3), 189-200.

(4) Guettler, J. H.; Demetropoulos, C. K.; Yang, K. H.; Jurist, K. A. Osteochondral Defects in the Human Knee Influence of Defect Size on Cartilage Rim Stress and Load Redistribution to Surrounding Cartilage. Am. J. Sports Med. 2004, 32 (6), 1451-1458.

(5) Vijayan, S.; Bentley, G.; Briggs, T.; Skinner, J.; Carrington, R.; Pollock, R.; Flanagan, A. Cartilage Repair: A Review of Stanmore Experience in the Treatment of Osteochondral Defects in the Knee with Various Surgical Techniques. Indian J. Orthop. 2010, 44 (3), 238.

(6) Bhardwaj, N.; Devi, D.; Mandal, B. B. Tissue-Engineered Cartilage: The Crossroads of Biomaterials, Cells and Stimulating Factors. Macromol. Biosci. 2015, 15 (2), 153-182.

(7) Mandal, B. B.; Park, S. H.; Gil, E. S.; Kaplan, D. L. Multilayered Silk Scaffolds For Meniscus Tissue Engineering. Biomaterials 2011, 32 (2), 639-651.

(8) Martin, I.; Miot, S.; Barbero, A.; Jakob, M.; Wendt, D. Osteochondral Tissue Engineering. J. Biomech. 2007, 40 (4), 750-765.

(9) Panseri, S.; Russo, A.; Cunha, C.; Bondi, A.; Di Martino, A.; Patella, S.; Kon, E. Osteochondral Tissue Engineering Approaches for Articular Cartilage and Subchondral Bone Regeneration. Knee Surg. Traumatol. Arthrosc. 2012, 20 (6), 1182-1191.

(10) Li, J. J.; Kim, K.; Roohani-Esfahani, S. I.; Guo, J.; Kaplan, D. L.; Zreiqat, H. A Biphasic Scaffold Based on Silk and Bioactive Ceramic with Stratified Properties for Osteochondral Tissue Regeneration. J. Mater. Chem. B 2015, 3 (26), 5361-5376.

(11) Kundu, B.; Rajkhowa, R.; Kundu, S. C.; Wang, X. Silk Fibroin Biomaterials for Tissue Regenerations. Adv. Drug Delivery Rev. 2013, 65 (4), 457-470.

(12) Singh, Y. P.; Bhardwaj, N.; Mandal, B. B. Potential of Agarose/ Silk Fibroin Blended Hydrogel for In Vitro Cartilage Tissue Engineering. ACS Appl. Mater. Interfaces 2016, 8 (33), 21236-21249.

(13) Bhardwaj, N.; Singh, Y. P.; Devi, D.; Kandimalla, R.; Kotoky, J.; Mandal, B. B. Potential of Silk Fibroin/Chondrocyte Constructs of Muga Silkworm Antheraea assamensis for Cartilage Tissue Engineering. J. Mater. Chem. B 2016, 4 (21), 3670-3684.

(14) Zhang, F.; Zuo, B.; Fan, Z.; Xie, Z.; Lu, Q.; Zhang, X.; Kaplan, D. L. Mechanisms and Control of Silk-Based Electrospinning. Biomacromolecules 2012, 13 (3), 798-804.

(15) Jones, J. R. Review of Bioactive Glass: from Hench to Hybrids. Acta Biomater. 2013, 9 (1), 4457-4486.

(16) Li, R.; Clark, A. E.; Hench, L. L. An Investigation of Bioactive Glass Powders by Sol-Gel Processing. J. Appl. Biomater. 1991, 2 (4), 231-239.

(17) Kim, H. W.; Kim, H. E.; Knowles, J. C. Production and Potential of Bioactive Glass Nanofibers as a Next-Generation Biomaterial. Adv. Funct. Mater. 2006, 16 (12), 1529-1535.
(18) Gupta, A.; Mita, K.; Arunkumar, K. P.; Nagaraju, J. Molecular Architecture of Silk Fibroin of Indian Golden Silkmoth, Antheraea assama. Sci. Rep. 2015, 5, 12706.

(19) You, R.; Xu, Y.; Liu, Y.; Li, X.; Li, M. Comparison of the In Vitro and In Vivo Degradations of Silk Fibroin Scaffolds from Mulberry and Nonmulberry Silkworms. Biomed. Mater. 2015, 10 (1), 015003.

(20) Rockwood, D. N.; Preda, R. C.; Yücel, T.; Wang, X.; Lovett, M. L.; Kaplan, D. L. Materials Fabrication from Bombyx mori Silk Fibroin. Nat. Protoc. 2011, 6 (10), 1612-1631.

(21) Mandal, B. B.; Kundu, S. A Novel Method for Dissolution and Stabilization of Non-Mulberry Silk Gland Protein Fibroin Using Anionic Surfactant Sodium Dodecyl Sulfate. Biotechnol. Bioeng. 2008, 99 (6), 1482-1489.

(22) Gil, E. S.; Kluge, J. A.; Rockwood, D. N.; Rajkhowa, R.; Wang, L.; Wang, X.; Kaplan, D. L. Mechanical Improvements to Reinforced Porous Silk Scaffolds. J. Biomed. Mater. Res., Part A 2011, 99 (1), 1628

(23) Gupta, P.; Kumar, M.; Bhardwaj, N.; Kumar, J. P.; Krishnamurthy, C.; Nandi, S. K.; Mandal, B. B. Mimicking Form and Function of Native Small Diameter Vascular Conduits Using Mulberry and Non-Mulberry Patterned Silk Films. ACS Appl. Mater. Interfaces 2016, 8.25, 15874-15888.

(24) Kaur, T.; Thirugnanam, A. Tailoring In Vitro Biological and Mechanical Properties of Polyvinyl Alcohol Reinforced with Threshold Carbon Nanotube Concentration for Improved Cellular Response. RSC Adv. 2016, 6 (46), 39982-39992.

(25) Rawadi, G.; Vayssière, B.; Dunn, F.; Baron, R. Roman-Roman, S., BMP-2 Controls Alkaline Phosphatase Expression and Osteoblast Mineralization by a Wnt Autocrine Loop. J. Bone Miner. Res. 2003, 18 (10), 1842-1853.

(26) Park, S. H.; Gil, E. S.; Mandal, B. B.; Cho, H. S.; Kluge, J. A.; Min, B.-H.; Kaplan, D. L. Annulus Fibrosus Tissue Engineering Using Lamellar Silk Scaffolds. J. Tissue Eng. Regener. Med. 2012, 6 (Suppl 3), s24-s33.

(27) Park, S.-H.; Gil, E. S.; Cho, H.; Mandal, B. B.; Tien, L. W.; Min, B.-H.; Kaplan, D. L. Intervertebral Disk Tissue Engineering Using Biphasic Silk Composite Scaffolds. Tissue Eng., Part A 2011, 18 (5-6), 447-458.

(28) Prein, C.; Warmbold, N.; Farkas, Z.; Schieker, M.; Aszodi, A.; Clausen-Schaumann, H. Structural and Mechanical Properties of the Proliferative Zone of the Developing Murine Growth Plate Cartilage Assessed by Atomic Force Microscopy. Matrix Biol. 2016, 50, 1-15.

(29) Schipani, E.; Ryan, H. E.; Didrickson, S.; Kobayashi, T.; Knight, M.; Johnson, R. S. Hypoxia in Cartilage: HIF- $1 \alpha$ is Essential for Chondrocyte Growth Arrest and Survival. Genes Dev. 2001, 15 (21), $2865-2876$

(30) Sherman, V. R.; Yang, W.; Meyers, M. A. The Materials Science of Collagen. J. Mech. Behav. Biomed. Mater. 2015, 52, 22-50.

(31) Peng, C.; Liu, P.; Hu, J.; Hua, T.; Shen, Y.; Zhao, B.; Tang, G. Preparation of Uniaxially Aligned Mullite Ceramic Fibers by Electrospinning. Colloids Surf., A 2014, 457, 1-7.

(32) Spalazzi, J. P.; Dionisio, K. L.; Jiang, J.; Lu, H. H. Osteoblast and Chondrocyte Interactions During Coculture on Scaffolds. IEEE Eng. Med. Biol. Mag. 2003, 22 (5), 27-34.

(33) Oliveira, J. M.; Rodrigues, M. T.; Silva, S. S.; Malafaya, P. B.; Gomes, M. E.; Viegas, C. A.; Dias, I. R.; Azevedo, J. T.; Mano, J. F.; Reis, R. L. Novel Hydroxyapatite/Chitosan Bilayered Scaffold for Osteochondral Tissue-Engineering Applications: Scaffold Design and its Performance when Seeded with Goat Bone Marrow Stromal Cells. Biomaterials 2006, 27 (36), 6123-6137.

(34) Angele, P.; Kujat, R.; Nerlich, M.; Yoo, J.; Goldberg, V.; Johnstone, B. Engineering of Osteochondral Tissue with Bone Marrow Mesenchymal Progenitor Cells in a Derivatized Hyaluronan-Gelatin Composite Sponge. Tissue Eng. 1999, 5 (6), 545-553.

(35) Hutmacher, D. W. Scaffolds in Tissue Engineering Bone and Cartilage. Biomaterials 2000, 21 (24), 2529-2543.

(36) Badami, A. S.; Kreke, M. R.; Thompson, M. S.; Riffle, J. S.; Goldstein, A. S. Effect of Fiber Diameter on Spreading, Proliferation, 
And Differentiation of Osteoblastic Cells on Electrospun Poly(Lactic Acid) Substrates. Biomaterials 2006, 27 (4), 596-606.

(37) Pham, Q. P.; Sharma, U.; Mikos, A. G. Electrospun Poly $(\varepsilon$ Caprolactone) Microfiber and Multilayer Nanofiber/Microfiber Scaffolds: Characterization of Scaffolds and Measurement of Cellular Infiltration. Biomacromolecules 2006, 7 (10), 2796-2805.

(38) Salinas, A. J.; Martin, A. I.; Vallet-Regí, M. Bioactivity of Three $\mathrm{CaO}-\mathrm{P}_{2} \mathrm{O}_{5}-\mathrm{SiO}_{2}$ Sol-Gel Glasses. J. Biomed. Mater. Res. 2002, 61 (4), 524-532.

(39) M. Mukundan, L.; Nirmal, R.; Vaikkath, D.; Nair, P. D. A New Synthesis Route To High Surface Area Sol Gel Bioactive Glass Through Alcohol Washing: A Preliminary Study. Biomatter 2013, 3 (2), e24288.

(40) Pereira, M. M.; Jones, J. R.; Orefice, R. L.; Hench, L. L. Preparation of Bioactive Glass-Polyvinyl Alcohol Hybrid Foams by the Sol-Gel Method. J. Mater. Sci.: Mater. Med. 2005, 16 (11), 1045-1050.

(41) Cacciotti, I.; Lombardi, M.; Bianco, A.; Ravaglioli, A.; Montanaro, L. Sol-Gel Derived 45S5 Bioglass: Synthesis, Microstructural Evolution and Thermal Behaviour. J. Mater. Sci.: Mater. Med. 2012, 23 (8), 1849-1866.

(42) Bhattacharjee, P.; Kundu, B.; Naskar, D.; Maiti, T. K.; Bhattacharya, D.; Kundu, S. C. Nanofibrous Nonmulberry Silk/PVA Scaffold for Osteoinduction and Osseointegration. Biopolymers 2015, 103 (5), 271-284.

(43) Peer, P.; Stenicka, M.; Pavlinek, V.; Filip, P. The Storage Stability of Polyvinylbutyral Solutions from an Electrospinnability Standpoint. Polym. Degrad. Stab. 2014, 105, 134-139.

(44) U.S. Food and Drug Administration. CFR Code of Federal Regulations, Title 21. http://www.accessdata.fda.gov/scripts/cdrh/ cfdocs/cfcfr/cfrsearch.cfm?fr=175.105 (accessed February 2017).

(45) Mansur, H. S.; Oréfice, R. L.; Mansur, A. A. Characterization of Poly (Vinyl Alcohol)/Poly (Ethylene Glycol) Hydrogels and PVADerived Hybrids by Small-Angle X-Ray Scattering and FTIR Spectroscopy. Polymer 2004, 45 (21), 7193-7202.

(46) Zhou, J.; Cao, C.; Ma, X.; Hu, L.; Chen, L.; Wang, C. In Vitro and In Vivo Degradation Behavior of Aqueous-Derived Electrospun Silk Fibroin Scaffolds. Polym. Degrad. Stab. 2010, 95 (9), 1679-1685.

(47) Amiraliyan, N.; Nouri, M.; Kish, M. H. Structural Characterization and Mechanical Properties of Electrospun Silk Fibroin Nanofiber Mats. Polym. Sci., Ser. A 2010, 52 (4), 407-412.

(48) Lai, G.-J.; Shalumon, K.; Chen, S. H.; Chen, J.-P. Composite Chitosan/Silk Fibroin Nanofibers for Modulation of Osteogenic Differentiation and Proliferation of Human Mesenchymal Stem Cells. Carbohydr. Polym. 2014, 111, 288-297.

(49) Wang, M.; Jin, H. J.; Kaplan, D. L.; Rutledge, G. C. Mechanical Properties of Electrospun Silk Fibers. Macromolecules 2004, 37 (18), 6856-6864.

(50) Arcos, D.; Greenspan, D.; Vallet-Regí, M. A New Quantitative Method to Evaluate the In Vitro Bioactivity of Melt and Sol-GelDerived Silicate Glasses. J. Biomed. Mater. Res., Part A 2003, 65 (3), 344-351.

(51) Bielby, R. C.; Christodoulou, I. S.; Pryce, R. S.; Radford, W. J.; Hench, L. L.; Polak, J. M. Time and Concentration Dependent Effects of Dissolution Products of $58 \mathrm{~S}$ Sol-Gel Bioactive Glass on Proliferation and Differentiation of Murine and Human Osteoblasts. Tissue Eng. 2004, 10 (7-8), 1018-1026.

(52) Acharya, C.; Ghosh, S. K.; Kundu, S. C. Silk Fibroin Film from Non-Mulberry Tropical Tasar Silkworms: A Novel Substrate for In Vitro Fibroblast Culture. Acta Biomater. 2009, 5 (1), 429-437.

(53) Lutolf, M.; Hubbell, J. Synthetic Biomaterials as Instructive Extracellular Microenvironments for Morphogenesis in Tissue Engineering. Nat. Biotechnol. 2005, 23 (1), 47-55.

(54) Malay, A. D.; Sato, R.; Yazawa, K.; Watanabe, H.; Ifuku, N.; Masunaga, H.; Hikima, T.; Guan, J.; Mandal, B. B.; Damrongsakkul, S. Relationships Between Physical Properties And Sequence in Silkworm Silks. Sci. Rep. 2016, 6, 27573.

(55) Shin, H.; Jo, S.; Mikos, A. G. Biomimetic Materials for Tissue Engineering. Biomaterials 2003, 24 (24), 4353-4364.
(56) Hersel, U.; Dahmen, C.; Kessler, H. RGD Modified Polymers: Biomaterials for Stimulated Cell Adhesion and Beyond. Biomaterials 2003, 24 (24), 4385-4415.

(57) Mandal, B. B.; Gil, E. S.; Panilaitis, B.; Kaplan, D. L. Laminar Silk Scaffolds for Aligned Tissue Fabrication. Macromol. Biosci. 2013, 13 (1), 48-58.

(58) Tew, S. R.; Murdoch, A. D.; Rauchenberg, R. P.; Hardingham, T. E. Cellular Methods in Cartilage Research: Primary Human Chondrocytes in Culture and Chondrogenesis in Human Bone Marrow Stem Cells. Methods 2008, 45 (1), 2-9.

(59) Puetzer, J. L.; Petitte, J. N.; Loboa, E. G. Comparative Review of Growth Factors for Induction of Three-Dimensional In Vitro Chondrogenesis in Human Mesenchymal Stem Cells Isolated from Bone Marrow And Adipose Tissue. Tissue Eng., Part B 2010, 16 (4), $435-444$.

(60) Kundu, B.; Kundu, S. C. Osteogenesis of Human Stem Cells in Silk Biomaterial for Regenerative Therapy. Prog. Polym. Sci. 2010, 35 (9), 1116-1127.

(61) Xynos, I. D.; Edgar, A. J.; Buttery, L. D.; Hench, L. L.; Polak, J. M. Gene-expression Profiling of Human Osteoblasts Following Treatment with the Ionic Products of Bioglass ${ }^{\circledR} 45 S 5$ Dissolution. J. Biomed. Mater. Res. 2001, 55 (2), 151-157.

(62) Jiang, J.; Nicoll, S. B.; Lu, H. H. Co-culture of Osteoblasts and Chondrocytes Modulates Cellular Differentiation In Vitro. Biochem. Biophys. Res. Commun. 2005, 338 (2), 762-770.

(63) Cao, T.; Ho, K. H.; Teoh, S. H. Scaffold Design and In Vitro Study of Osteochondral Coculture in a Three-Dimensional Porous Polycaprolactone Scaffold Fabricated by Fused Deposition Modeling. Tissue Eng. 2003, 9 (4,Suppl. 1), 103-112.

(64) Ma, Z.; Gao, C.; Gong, Y.; Shen, J. Chondrocyte Behaviors on Poly-L-Lactic Acid (PLLA) Membranes Containing Hydroxyl, Amide or Carboxyl Groups. Biomaterials 2003, 24 (21), 3725-3730.

(65) LiVecchi, A.; Tombes, R.; LaBerge, M. In Vitro Chondrocyte Collagen Deposition Within Porous HDPE: Substrate Microstructure and Wettability Effects. J. Biomed. Mater. Res. 1994, 28 (8), 839-850.

(66) Loeser, R. F. Chondrocyte Integrin Expression and Function. Biorheology 2000, 37 (1, 2), 109-116.

(67) Midha, S.; Murab, S.; Ghosh, S. Osteogenic Signaling on SilkBased Matrices. Biomaterials 2016, 97, 133-153.

(68) Vishwakarma, A.; Bhise, N. S.; Evangelista, M. B.; Rouwkema, J.; Dokmeci, M. R.; Ghaemmaghami, A. M.; Vrana, N. E.; Khademhosseini, A. Engineering Immunomodulatory Biomaterials to Tune the Inflammatory Response. Trends Biotechnol. 2016, 34 (6), $470-482$.

(69) Meinel, L.; Hofmann, S.; Karageorgiou, V.; Kirker-Head, C.; McCool, J.; Gronowicz, G.; Zichner, L.; Langer, R.; Vunjak-Novakovic, G.; Kaplan, D. L. The Inflammatory Responses to Silk Films In Vitro and In Vivo. Biomaterials 2005, 26 (2), 147-155. 